\title{
A Lean, Fast Mars Round-trip Mission Architecture: Using Current Technologies for a Human Mission in the 2030s
}

\author{
Lora Bailey, \\ NASA JSC, Houston, TX, 77058, USA
}

David Foltał Brent W. Barbee NASA GSFC, Greenbelt, MD, 20771, USA

\author{
Bruce Campbell, \\ ATK Spacecraft Systems, Beltsville, MD, 20705, USA \\ Harley Thronson,** \\ NASA GSFC, Greenbelt, MD, 20771, USA \\ and \\ Tzu Yu Lin ${ }^{\dagger \dagger}$ \\ University of Florida, Gainesville, FL, 32611, USA
}


We present a lean fast-transfer architecture concept for a first human mission to Mars that utilizes current technologies and two pivotal parameters: an end-to-end Mars mission duration of approximately one year, and a deep space habitat of approximately 50 metric tons. These parameters were formulated by a 2012 deep space habitat study conducted at the NASA Johnson Space Center (JSC) that focused on a subset of recognized highengineering-risk factors that may otherwise limit space travel to destinations such as Mars or near-Earth asteroid (NEA)s. With these constraints, we model and promote Mars mission opportunities in the 2030s enabled by a combination of on-orbit staging, mission element pre-positioning, and unique round-trip trajectories identified by state-of-the-art astrodynamics algorithms.

\section{Introduction}

\section{I.A. Overview}

W e describe a lean, reduced-duration round trip to Mars, which uses current technology with modest, reasonable engineering advancements where needed. We expect that development of a a round trip to Mars that is approximately one year in duration, using a modest crew vehicle size and mass, which produces conditions of reasonable risk that can be mitigated within the scope of conventional or practical engineering designs. We believe that this strategy holds the key which promotes confidence that such a venture can be realized in the 2030s.

Current NASA Design Reference Missions (DRMs) to Mars that are planned for the 2030s have largely been formulated around a number of significant technology advancements such as Nuclear Thermal Propulsion (NTP), long-duration cryogenic hydrogen storage, and in-situ resource utilization. ${ }^{1}$ However, progress to date of such capabilities has been limited in funding and development, and these will require lengthy, costly, and challenging research and development efforts.

These DRMs also last several hundred $(\sim 900+)$ days and thus require nontrivial overhead and risk planning measures that must be accommodated in the crew transit vehicle as well

\footnotetext{
*Aerospace Engineer, Code EA32, 2101 NASA Parkway, AIAA Senior Member.

${ }^{\dagger}$ Aerospace Engineer, Navigation and Mission Design Branch (Code 595), and AIAA Member Grade.

$\ddagger$ Aerospace Engineer, Navigation and Mission Design Branch (Code 595), AIAA Member.

$\S$ Aerospace Engineer, Navigation and Mission Design Branch (Code 595).

ฯAerospace Engineer, Systems Engineering Services and Advanced Concepts Branch (Code 592).

"Senior Systems Engineer, ATK Space Systems Division, AIAA Senior Member.

**Senior Scientist for Advanced Concepts, Code 660, AIAA Member.

${ }^{\dagger}$ Graduate Student, Mechanical and Aerospace Engineering Department, AIAA Student Member.
} 
as on the planetary surface. However, this in turn, inflates the mass and volume of the vehicle that provides the habitation and transit for the crew's journey. Moreover, there is considerable risk for crew health and performance due to lengthy exposure to radiation and microgravity that are not well understood, but must also be addressed with risk mitigation and countermeasures in this undertaking.

Therefore, it seemed prudent that we begin to investigate a Mars mission architecture that significantly shortens the mission duration and requires only modest technology advancements that are well within practical reach, in order to create the opportunity for reasonable engineering design solutions and the possibility of travel to Mars by the 2030s. Additionally, this offers significant relief to crew health and performance risks as well as reducing the potential overhead of mass and volume penalties to the transit vehicle, thus enabling a lighter payload. We expect that by creating a short Mars excursion that is limited to approximately a one-year round trip, the engineering risk controls for managing the crew and vehicle become feasible, and we have a mission that supports engineering plausibility in the 2030s.

This study was initiated by the Deep Space Habitat (DSH) Project at the JSC in October, 2011 to provide a more in-depth evaluation of a general deep space transit vehicle for longduration, remote missions without a specific destination. ${ }^{2}$ Our intent was to focus on transit vehicle design to reduce the significant engineering challenges of deep-space travel such as galactic cosmic radiation (GCR) and vehicle reliability, but also to promote as lightweight and lean a design as possible in terms of vehicle mass, power, and volume. Our study of the deep-space engineering challenges was focused on three main areas: 1) GCR, 2) vehicle reliability with emphasis on life-support, and 3) crew health and performance. In conducting these studies, we concluded that all of these (and other) high-risk factors that hinder deep space travel are significantly reduced by one common risk control: limiting the round trip to approximately one year.

As a result, the prospect of a one-year round trip to Mars was then initiated and investigated by the DSH project ${ }^{3}$ that emphasized use of conventional chemical propulsion using the "Fast Mars Transfer" (FMT) technique developed by Folta et. al. ${ }^{4,5}$ A preliminary assessment using Fast Mars Transfer (FMT) was conducted, which provided preliminary analysis and architecture definition by November 2012. We expected that this preliminary analysis would provide data to help determine concept feasibility in our study as well as identify specific areas where there were advantages or needs for more in-depth analysis. It is both the studies of the deep-space engineering challenges, as well as the results from the subsequent FMT assessment, which are the subjects of this paper. 


\section{I.B. Background}

The DSH Project is an Advanced Exploration Systems (AES) project that is led at the JSC. This paper is the result of a JSC DSH effort thatwas informally begun in October, 2011 to develop a design for a generic deep-space transit vehicle that was to:

1. be manned for a minimum of one full year without crew change

2. utilize a launch packaging plan around commercial expendable launch vehicles (ELVs) in addition to the Space Launch System (SLS) heavy lift vehicle

3. focus on priority engineering challenges to deep-space travel, and provide a viable concept that addresses them

This ultimately resulted in a 2012 design for a first mission to Mars that uses current or near-future technologies to solve the engineering challenges that currently hinder such a venture. Current NASA Mars mission architectures ${ }^{1}$ are primarily based on long-duration stays on the Mars surface, which require equipment and supplies to be pre-launched to the Mars surface before launching the crew to the planet.

These NASA missions are notably over 900-day trips, which in some cases include a 500-day orbit around Mars waiting for a favorable alignment of Earth and Mars to manage and reduce the fuel cost of the return. These mission scenarios require approximately 900 days or more of supplies that include maintenance items, repair capabilities, water, food, and spares. There are also significant risks for crew health and well-being for these lengthy durations in space, as well as vehicle reliability concerns in an isolated, remote destination. Moreover, these Mars missions propose to use currently undeveloped high-efficiency nuclear thermal propulsion and power systems, which also requires an additional development of long-duration storage of cryogenic liquids to preclude immediate boil-off loss of liquid hydrogen. In addition, these missions may require a Venus flyby to assist the return of the crew vehicle, which can amplify radiation exposure and thermal heat rejection and power demands on the vehicle for passing through an area that is approximately 0.7 AU from the Sun. These are all concerns that can be significantly mitigated and/or disregarded altogether based on the conclusions of our investigation.

We also note, however, that the effort to date in this feasibility investigation necessitates

additional studies and/or more in-depth analyses. Our suggested follow-on analyses are described further at the conclusion of this paper. 


\section{Approach}

A three-prong approach ${ }^{2}$ was formulated to initiate the definition and study of a vehicle concept for a 365-day manned mission that could be launched in packages limited to the upmass and fairing limitations of expendable launch vehicle (ELV)s. This approach included the following three-prong assessments that were executed in parallel where possible: definition of 1) transit vehicle requirements, 2) top engineering risks and mitigation, and 3) launch/transit and operations.

In beginning the effort to define and evaluate a deep-space vehicle concept that meets the intent of the three intended goals, we defined some self-prescribed top-level requirements. Our definition of these requirements, albeit artificial, was intended to be reasonable and well-anchored, and was used to provide a solid basis for assessment of valid engineering design, environments, and operations. We generated requirements in cooperation with a subset of members from NASA JSC's Human Architecture Team (HAT), in order to ensure commonality of purpose and strategy for human architecture development within the agency.

From among the few Level Zero (agency) requirements that were generated from this activity, we note the following:

1. A human presence shall be established on the surface of Mars, in Mars orbit, or on a Mars moon within the time-frame of the next three decades.

2. A precursor mission or missions shall be performed, which establishes a human presence in deep space first at a cis/translunar location, and/or subsequently to other deep-space locations to serve as an incremental development, demonstration, and accumulation of progressing capability (towards human presence at Mars).

And among the Level 1 (vehicle) requirements that were generated, we note the following:

1. The purpose of the first deep- space vehicle is to act as a test bed for long-duration deep-space (outside the Van Allen belt) evaluations and technologies, and as such, shall contain the architecture, elements, environmental engineering, and features that will serve to substantively vet and verify its acceptability as a Mars-mission transit vehicle.

2. The location of the first deep-space vehicle shall be EML1 or EML2 due to its reasonable proximity to Earth that provides a rapid capability for crew return or other emergency or non-emergency provisioning and repairs, as well as favorable cost and weight for both station-keeping and delta-v for transit.

With our newly defined requirements and three initially-defined goals, we found merit in adopting a recently-produced transit vehicle concept to study that was serendipitously 
well-matched with these goals and requirements. This transit vehicle had been previously produced by the DSH project in fiscal year 2011 at the National Aeronautics and Space Administration (NASA) Marshall Space Flight Center (MSFC) as a transit vehicle to accommodate a 500-day mission with a crew of four. The vehicle concept, shown in Figure 1, is known as the "International Space Station (ISS)-Derived Concept," ${ }^{6}$ because it contained ISS modules and architectural elements that were available assets for NASA use as shown in Figure 2. These modules and architectural elements then could be, by definition, employed for expeditious deep-space use, and they could be evaluated for ELV launch mass, volume, and environments as part of the initial goals assessment. This vehicle concept was welldeveloped and serendipitously well-suited for the intended goals and our requirements in our new FY12 365-day study. By choosing a vehicle concept that had already been produced, we could begin our analyses of the vehicle immediately that addresses the engineering risks mentioned in prong 2. This would capitalize on what is known and available to date to exploit the use of well-established ISS modules, capabilities, models, and/or knowledge base. Moreover, it is prudent that we begin conducting deep-space evaluations of our conventional architectures and systems first in order to examine what is readily available before venturing into new vehicle designs which inherently contain new risks and unknowns.

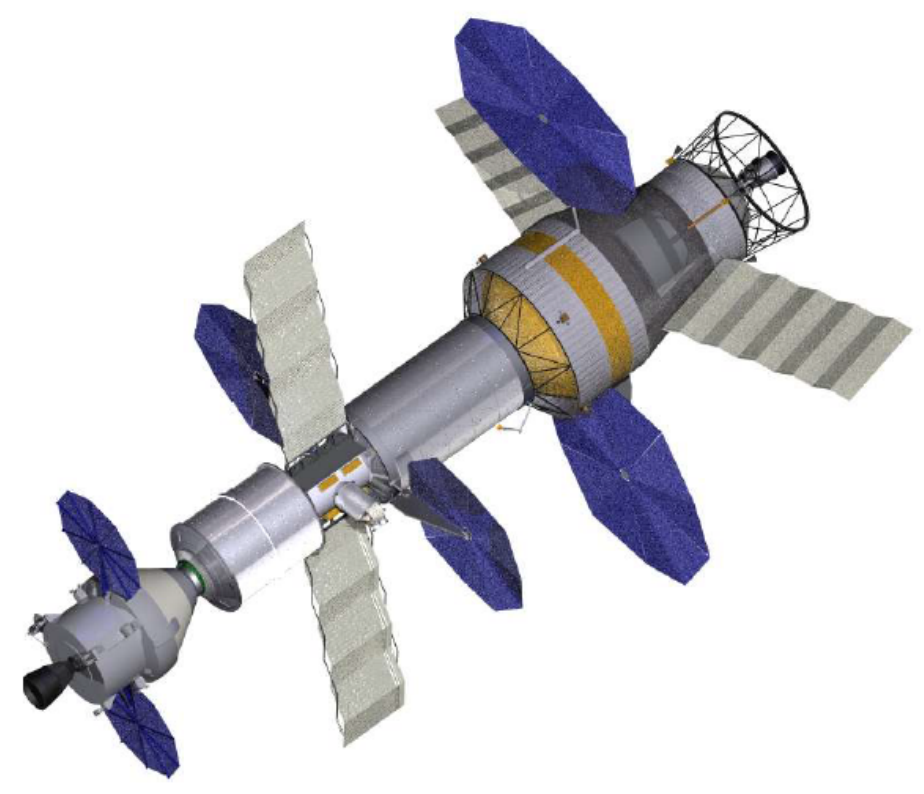

Figure 1. ISS-Derived Deep Space Transit Vehicle Concept ${ }^{6}$

As a caveat, however, our use of this vehicle concept is not intended as an endorsement of it as the "best" architecture for deep-space transit, but rather, that it offers a good starting point for a first assessment of engineering risks (i.e. GCR, reliability, etc.) using models and data that already exist.

The MSFC ISS-Derived concept has three core elements, mated in series as shown in 

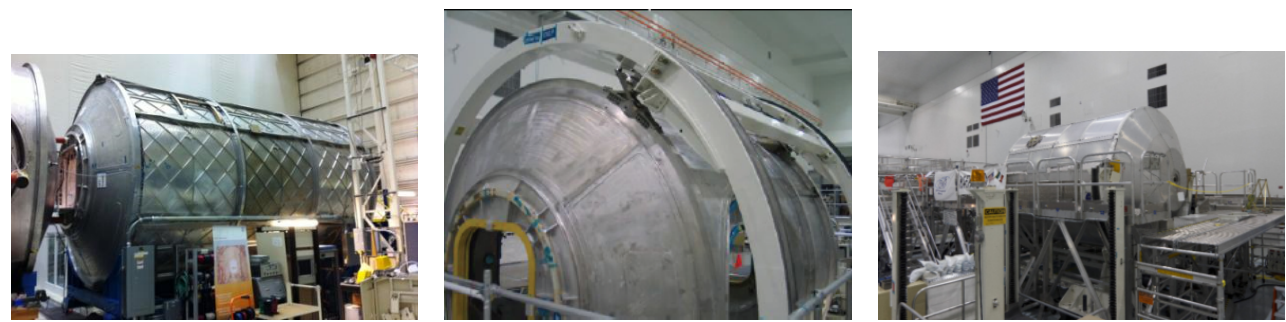

Figure 2. ISS Module Ground Assets Proposed for Flight

Figure 3: an ISS lab module, a generic tunnel/airlock, and an ISS Multi-Purpose Logistics Module (MPLM). It is powered and controlled by a general propulsion module that is docked on one end, and the crew is brought on board with an Orion capsule that is docked at the opposing end as shown in Figure 1.

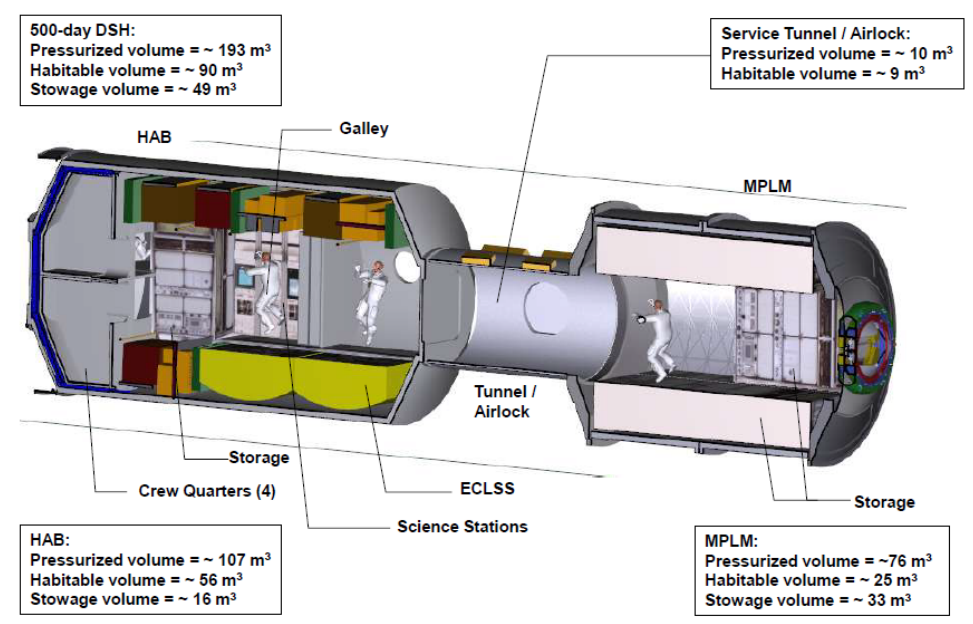

Figure 3. ISS-Derived Concept Core Habitable Volume

The MSFC ISS-Derived study utilized the three core modules as the working and living space, with Orion remaining essentially unpowered and dormant during the mission transit period and was primarily only intended to be used for crew arrival and Earth return re-entry. The core habitable volume was designed to accommodate four crew members and included the estimated logistics and spares for a generic 500-day transit mission. Although this is not precisely a 365-day vehicle concept that matches our initial study goals, it is similar to that expected of a one-year transit vehicle in terms of required habitable volume and overall subsystems and logistics. Moreover, since only best-estimate approximations are used in such concept studies, we accepted the additional mass that may or may not exist in this concept with regard to to its predicted food, power, and any supplies to accommodate 500 days rather than precisely 365 days, since this could serve as a reserve for inaccuracies or unknowns in our own preliminary assessment. 


\section{Top Engineering Risks Definition, Assessment, and Mitigation Measures}

\section{III.A. Overview}

We next assessed the the second prong in the strategy of the study. That is, the definition, assessment, and mitigation of some of the top engineering risks to deep-space travel. Three of the top risks to deep-space transit that we investigated were: 1) GCR, 2) vehicle reliability, with focus on environmental control and life support system (ECLSS) reliability, and 3) crew health and performance.

\section{III.B. Radiation Studies and General Information}

The first risk, GCR, can be viewed as the greatest challenge to remote deep-space transit and exploration. Without addressing this challenge, we are currently not at liberty to conduct deep space transit and exploration outside the Van Allen belt for durations longer than approximately 180 days. Our intent, then, is to begin by pursuing solutions that implement a smart architecture strategy. We do so by evaluating our current ISS elements, incorporating little/no parasitic mass shielding, and discovering where we can meet requirements in moderation (not necessarily for the worst case) by addressing crew and vehicle protection via a combination of reasonable technological and operational solutions.

We began with a vehicle radiation analysis to assess the flight-worthiness of the MSFC ISS-Derived Concept when exposed to 365 consecutive days of GCR. General informationgathering about radiation was part of our initial study effort, and we include such discussion here support for the rationale used in the vehicle radiation analysis and final conclusions of this study. We start by noting the two basic categories of deep space radiation: 1) solar particle events (SPE) and 2) GCR. SPE can simply be described as lower-energy particles (compared to GCR) resulting primarily from solar flares and coronal mass ejections. These solar particle events occur occasionally, but infrequently, and are readily monitored to predict the potential ISS crew radiation exposure. Solar activity has an 11-year solar cycle, during which time SPEs occur most significantly during the "solar maxima" period of the solar cycle. SPE radiation exposure to the ISS crew, for example, is mitigated by reporting any predicted unacceptably high radiation at the time of the event and having the crew seek shelter for that short period in a storm shelter on the space station.

GCR, however, contains significantly higher-energy particles and is continuous. For GCR, the temporary storm shelter offers no real mitigation for two significant reasons: a) the "temporary shelter" would essentially have to be the entire vehicle since the particle activity occurs continuously, and b) the use of protective sheltering/shielding is virtually ineffective 
due to the high energy of the particles involved. GCR is significantly mitigated for low-Earth orbit vehicles such as the ISS due to the Earth's protective magnetic field. However, for space vehicles traveling outside the Van Allen Belts, there is direct exposure to these destructive particles.

Figure $4^{7}$ shows the effectiveness of the radiation protection provided by a space vehicle as its hull thickness increases. This particular figure shows the effective radiation dose experienced by a male crew member that is exposed to deep-space GCR for a one-year period and who is positioned at the center of an idealized sphere with a variable hull thickness.

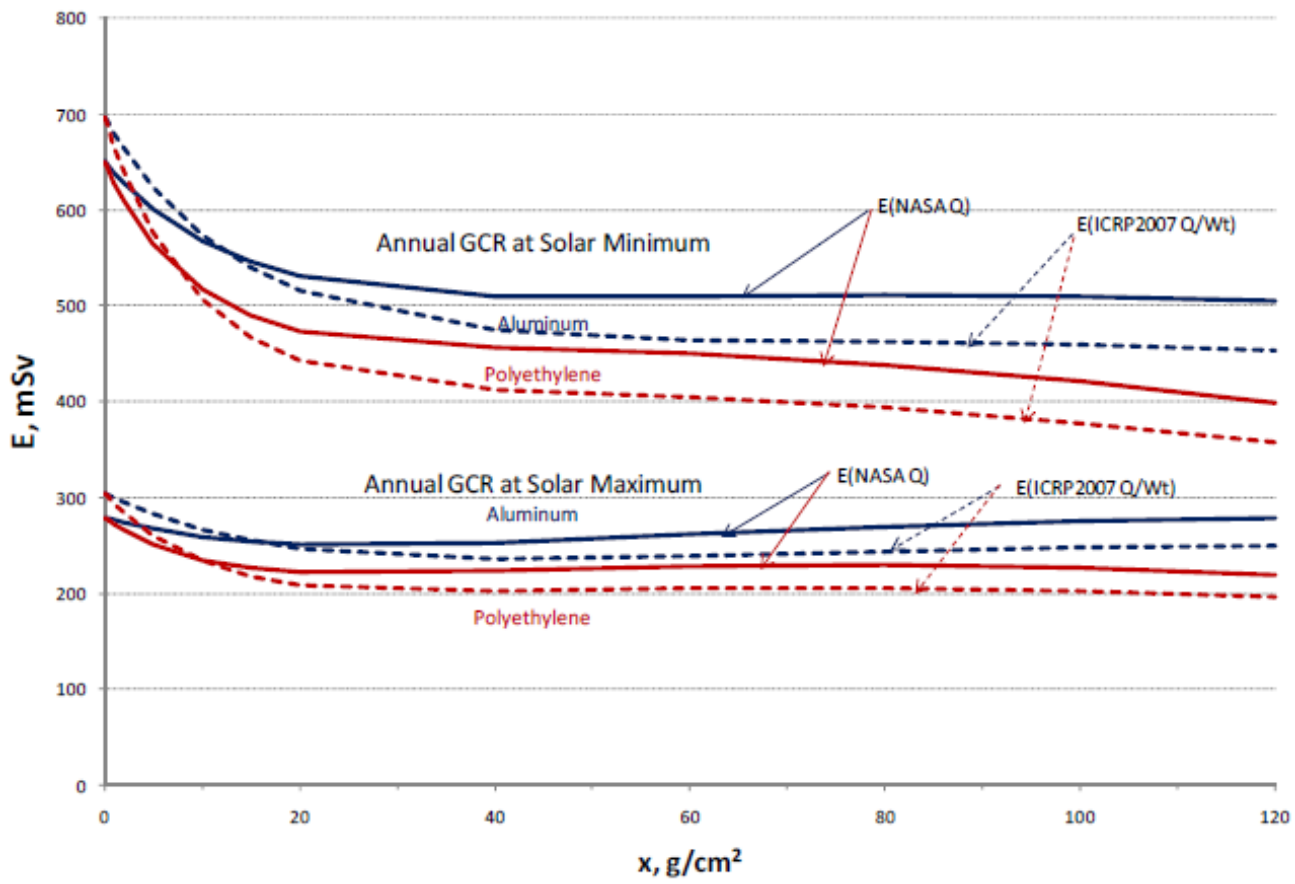

Figure 4. Effective GCR Dose for Males behind Shielding in One Year, with Effect of Solar Cycle ${ }^{7}$

Figure 4 also shows that there is a marked difference in crew radiation dose dependent upon when the GCR exposure occurs in a given solar cycle. Specifically, the GCR exposure is significantly reduced during a solar maximum as compared to during a solar minimum. For the cases shown in Figure 4, a crewman experiences about half the radiation dose from GCR during a solar maximum versus a solar minimum for a typical effective shielding mass of $20 \mathrm{~g} / \mathrm{cm}^{2}$. This value is comparable shielding to that offered from a typical ISS module. Note, however, that not every solar cycle behaves identically in that there are significant variations in solar maxima from cycle to cycle that may not produce consistent high levels of solar activity. Conversely, there are also variations in solar minimum behavior in that not every solar minimum shows consistently extremely low solar activity. As such, there are a group of curves that fill the "medium" in between these curve values that represent lower solar maximums and higher solar minimums than shown in this particular chart. Also of 
note, the solid lines in the chart represent the more current NASA model results.

In addition, we note that there is a difference in protection performance offered by a typical aluminum hull versus one constructed with polyethylene. Due to the lack of structural strength performance (and other material properties) of polyethylene as compared to aluminum or other materials, polyethylene has been used traditionally as an internal add-on feature with essentially no structural of "shared" purpose other than radiation protection. As such, it is usually used as purely supplemental mass that adds a shield in localized areas. Also note that the improvement in radiation dose reduction offered by polyethylene, relative to that offered by aluminum, is slightly better during a solar minimum than during a solar maximum.

Finally, we particularly note from Figure 4 the flatness of the curves at hull thicknesses heavier than about $20 \mathrm{~g} / \mathrm{cm}^{2}$. This implies that no real additional protection is gained when adding more shelter thickness/mass beyond approximately $20 \mathrm{~g} / \mathrm{cm}^{2}$, unless it is specifically a polyethylene or other densified-hydrogen form of material (water or cryogenic hydrogen). Water acts similarly (and masses comparably) to the performance of polyethylene curves. Cryogenic hydrogen (not shown) offers dramatic reduction in protective effectiveness and does so with a much lighter mass penalty. However, despite its lighter weight, the use of cryogenic hydrogen would incur a significant volume disadvantage, in addition to not being as human-friendly as water or polyethylene with regard to in-flight risks and hazards. Moreover, experiments of in-space active cooling for actual long-duration storage and use of cryogenic hydrogen are still in an early stage. Therefore in-space cryogenic hydrogen does not appear to be a feasible near-term capability for use in this application. We fully support, of course, that in-orbit storage of cryogenic hydrogen be developed for many reasons and certainly could offer significant radiation dose reduction as a shared benefit to any other application it may serve.

There are few radiation exposure requirements for space travel. We note two from NASASTD-3001 ${ }^{8,9}$ 1) the As Low As Reasonably Achievable (ALARA) principle, and 2) not exceeding the $3 \%$ increase to human risk of exposure-induced death (REID) at a $95 \%$ confidence level (CL). The latter is currently used for determining crew radiation exposure limits for low-Earth orbit applications, but wwas also used herein as an approximation to radiation limits for crew in deep-space applications.

There is debate that this requirement is too stringent to be practically applied for deepspace applications since radiation is likely to be less hazardous than other risks in deep-space or for space travel in general. For example, the crew risk of death due to a launch alone could be viewed as greater than the $3 \%$ increased risk of death due to radiation exposure. However, the radiation risk engenders an entirely different risk category than other risks traditionally addressed for space flight. Primarily, radiation risk is related to a potential 
death occurring later in life, well after the crewmember has been removed from the risk exposure. In the case of launch, however, the crew risk of death ceases to exist at the time of completion of a successful launch, whereas the unknown early risk of death is carried throughout the remaining life of a crewmember long after the exposure. This additionally includes the increased risk of experiencing and surviving various cancers or Alzheimer's disease, etc. during the crewmember's remaining living years. There are also other less known and unknown affects due to radiation exposure that are not included in the analysis applied to the 3\% REID, which itself is more focused on cancer-induced death from radiation as applies to blood-forming organs, skin exposure, etc.. We also note that the average number of years of life lost per death for an exposure-induced cancer (using the exposure limit of $3 \%$ REID) is approximately 12-16 years less for males and females who range from 25 to 55 years in age during the radiation exposure period. ${ }^{10}$ Therefore, there cannot be a cavalier approach in proposing a relief of the $3 \%$ REID as a requirement, nor an equivalency of proposed approach to launch risk mitigation and other such space hazards that no longer exist after the hazard is removed.

For this study we use 3\% REID at a 95\% CL. However we expect that a sensitivity study can also be performed to understand the variance created in crew dose for small changes in REID (varied from 3-5\% for example), or changes in CL (varied from 80-95\%). However, due to limited resources at the time of our study, this has yet to be performed.

We also began our study with a fuller understanding of the implications of permissible human radiation exposure limits. Figure $5^{10}$ shows an example of possible crew permissible exposure limits (PEL) based on no greater than a 3\% increase in REID at a 95\% CL. These data serve as an example only, but give an indication of the general dose limitations based on gender and age. For example, a 40-year-old male is estimated to have an approximately 400 milli-Sievert $(\mathrm{mSv})$ total radiation dose limit, whereas a 40-year-old female is estimated to have an approximately total $250 \mathrm{mSv}$ limit. This serves to show an approximation of career dose limits for crew and that males in general have a higher radiation tolerance compared to their female counterparts of the same age.

We note that among the history of missions ${ }^{10}$ ranging from Mercury, Gemini, Apollo, Skylab, Space Shuttle, Mir, and ISS, the doses of individual astronauts were almost all below approximately $5 \mathrm{mSv}$ per mission, with only a limited number of exposures occurring in the range from 10 to $100 \mathrm{mSv}$ per crewmember in a given mission aboard Mir and/or ISS. These exposures, of course are well below the crewmember PEL values noted from the approximations in the example case shown in Figure 5, although some are approaching limits that are of more concern, i.e. those closer to $100 \mathrm{mSv}$. 


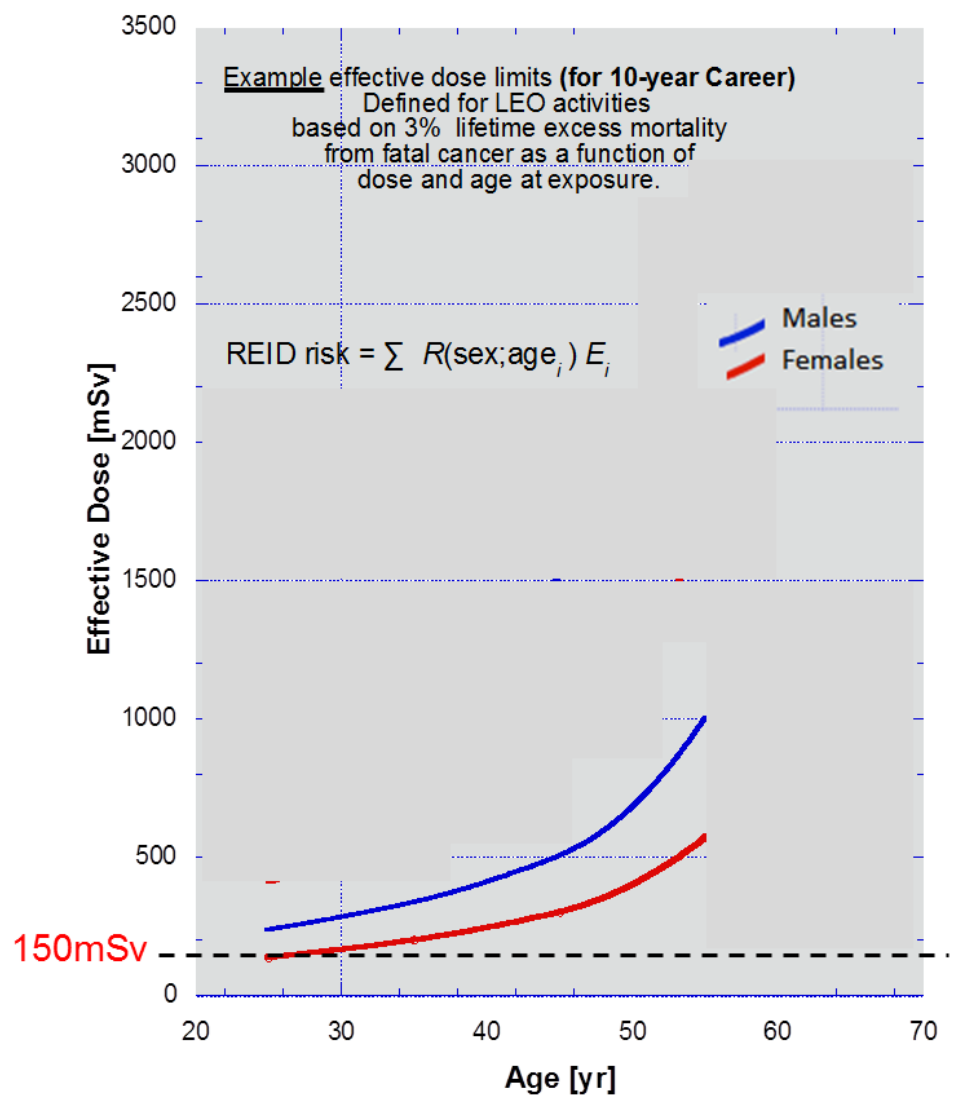

Figure 5. Example of Permissible Exposure Limits with Stochastic Effects ( 3 3\% REID at 95\% CL) ${ }^{10}$

\section{III.C. Radiation Shelter Materials Study}

Calculations ${ }^{11}$ were performed to show the necessary thickness required to gain an additional $10 \mathrm{~g} / \mathrm{cm}^{2}$ of effective radiation shielding using four different materials: aluminum, polyethylene, water, and liquid hydrogen. Results showed that in order to acquire an additional 10 $\mathrm{g} / \mathrm{cm}^{2}$ of protective shielding around a vehicle, it would require a $3.7 \mathrm{~cm}(1.5 \mathrm{in})$ thickness of aluminum, or a $10 \mathrm{~cm}$ (4 in) thickness of polyethylene or of water (polyethylene and water have the same density), or nearly a $142 \mathrm{~cm}$ (56 in) thickness of liquid hydrogen. Also, separate calculations were made that computed the weight burden of adding only $10 \mathrm{~g} / \mathrm{cm}^{2}$ of shielding around just one ISS lab module. That mass burden was computed to be 15,000 $\mathrm{kg}$, to add just $10 \mathrm{~g} / \mathrm{cm}^{2}$ of shielding to one lab-size module. This of course does not account for the mass penalty to an entire vehicle, but only for just one lab-sized module.

\section{III.D. Radiation Analysis of the ISS-Derived Concept}

An analysis ${ }^{12}$ was conducted by the NASA Space Radiation Analysis Group (SRAG) that evaluated the ISS-Derived Concept architecture for a one-year deep-space exposure to GCR. The analysis that was performed utilized high-fidelity module models to approximate the 
ISS-Derived Concept configuration and architecture by connecting the ISS Lab, Crewlock, and Node 2. ISS outfitting both interior and exterior to those modules remained unchanged from its ISS configuration as we considered it very similar to the outfitting for the ISSDerived Concept to within reasonable approximation for our purposes. The analysis utilized the 1977 Solar Minimum conditions for a one-year exposure and computed effective dose for a crew member at five different locations inside the vehicle. This resulted in a range of internal dosage from $394 \mathrm{mSv}$ to $456 \mathrm{mSv}$, and produced the risk of exposure-induced death from cancer due to radiation exposure as shown in Figure 6.

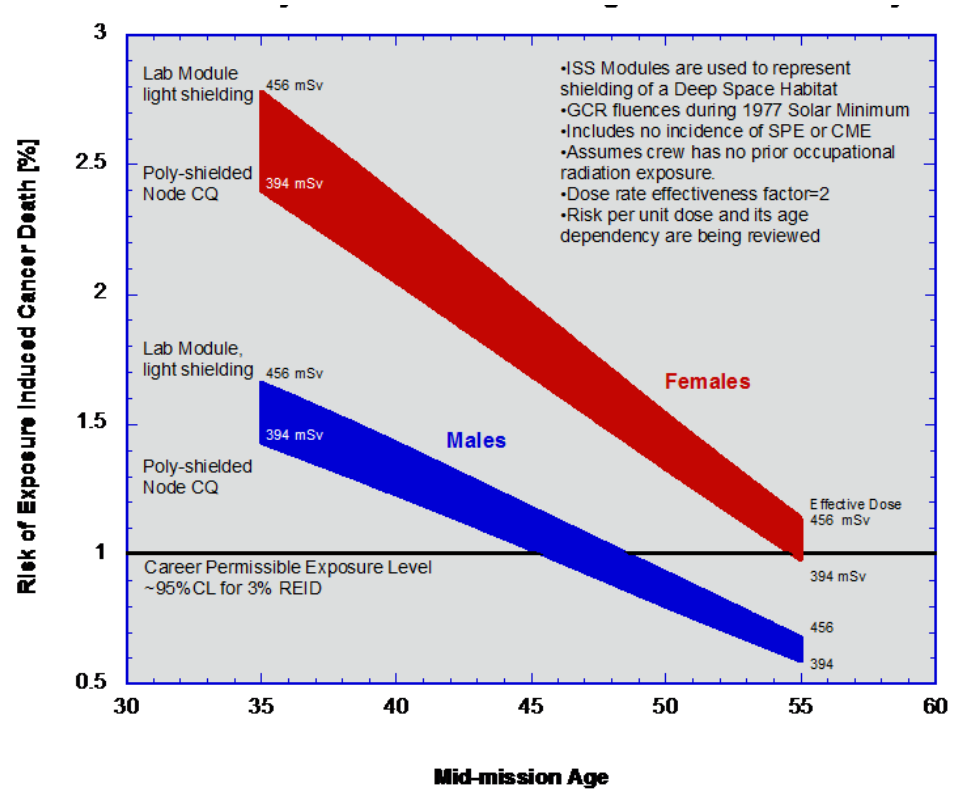

Figure 6. Increased Risk of Exposure-Induced Death ${ }^{13}$

The bars in the chart have a width equivalent to the range of dose between 394 to 456 $\mathrm{mSv}$, and are shown for males and females separately over the given age range. The black line represents the limit of the $3 \%$ REID at a 95\% CL, and suggests that males about 47 years old or older or females about 57 years old or older could be in range for a one-year mission at solar minimum, a likely worst case. We note that these doses are approximations for the purposes of our study and do not include SPE contributions. However, these SPE contributions to dose are much less in comparison to those from GCR. Furthermore, since the GCR doses are treated as linearly additive over time, we could also conclude that if a mission to a near-Earth object (NEO) or Mars could take perhaps 500 days, multiplying these dose values of 394 and $456 \mathrm{mSv}$ by a factor of 500/365 produces much higher dose values that would be out of permissible crew age range for meeting the $3 \%$ REID at $95 \%$ CL. Also, note that this would represent one mission's radiation exposure per crewmember and, therefore, would support selection of crewmembers having little or no previous in-space flight experience since this one mission would be near or at the lifetime/career PEL. 
Conversely, we note the favorable possibilities that GCR exposure may not be so severe as the 1977 Solar Minimum case and, that if crew travel occurs during a solar maximum, there is even greater probability of advantageous relief. Also, because crew radiation limitations are gender-and age-dependent, crew selection can be a contributing part of the risk mitigation strategy.

A typical ISS module, which possesses approximately $20 \mathrm{~g} / \mathrm{cm}^{2}$ of effective shielding, is already a highly efficient architecture in that greater amounts of shielding beyond that thickness provides little dose reduction in return for the mass increase. However, discrete amounts of polyethylene in localized areas or small shields that could be mobile with the crew member could supply shielding that may offer some modest dose reduction as a crew option if there is radiation that is more significant during a given trip than predicted.

However, two primary drivers of GCR exposure are a) solar cycle activity at the time of mission and b) duration of travel. As such, even if a deep-space mission occurs during a particular solar maximum which happens to offer significant GCR exposure reduction, but the overall trip duration is 900 days, this still fails to control radiation exposure to a limit of control limit of 3\% REID at a 95\% CL. Therefore, a separate contributor in balancing this equation is whether to dismiss the $3 \%$ REID at a 95\% CL and promote some other value as an acceptable risk, or, explore the possibility of reducing space-travel durations to well below $900+$ days in addition to using solar cycle "space weather" as a promising advantage to reduce GCR. We chose to pursue the latter, in a simple effort of attempting to keep crew and vehicle radiation exposure as low as possible.

In doing so, our primary purpose was a best-effort pursuit toward meeting the intent of NASA-STD-3001 requirement 4.2.10.5: In-flight radiation exposures shall be minimized using the ALARA principle. ${ }^{8}$

\section{III.E. Vehicle Reliability and Crew Health and Performance Studies}

Two other engineering risks to deep-space travel that were evaluated during our study were a) vehicle reliability, and b) crew health and performance. We did not carry out substantial new analysis in these areas, but largely reviewed the analyses and findings that already existed along with accompanying lessons learned.

We requested additional assessment to be performed that showed the greatest reliability risk issues for ISS. These reliability risks were assessed for their applicability to remote, deep-space travel, in contrast to Low Earth Orbit (LEO) risks that are readily addressible by sending up repairs, spares, new parts, or bringing the crew home in the event of a health concern or other emergency. ISS risks ${ }^{14}$ that correspond to subsystem reliability overall appear to be generally well-mitigated and general vehicle risks shared with a remote deepspace vehicle, with the exception of some involving crew health and in-flight medical care. 
Separately, there are the additional reliability risks for the deep-space application that had no need to be addressed by ISS in LEO, including the effects of GCR on the vehicle and crew, as well as much longer-duration microgravity effects on the crew.

Our reliability risk investigation results revealed direct correlations with the separate activity of researching and assessing risks associated with crew health and performance. These in turn proved to be interconnected with radiation exposure-induced crew health and in-flight performance risks. To name a few, risks associated with crew health and performance for a long-duration remote mission readily included radiation concerns, to include in-flight and post-flight cognitive and neural effects, as well as post-flight risk of cancer or death due to cancer. Moreover, there is considerable risk (known and unknown) associated with long-duration human exposure to microgravity for spaceflight assignments such as 900 day missions, and also serves as a serious strategic knowledge gap for in-flight and post-flight human health and performance. Treatment for medical issues and emergencies for the remote space travel application presents a substantial change in risk mitigations and considerations from our traditional ISS LEO controls for human health and performance. Other issues such as food packaging and storage to retain nutritive content beyond approximately 18 months is an additional critical risk which is still in research and development, since utilizing vitamin supplements cannot serve as a control due to the similar losses of its nutritive content in that same time period. As such, without a reasonable solution for preserving nutritive content, crew-members on missions lasting several hundred days could experience health issues not seen since early sea travel, such as scurvy.

Overall, the top three engineering risks that we evaluated during the study each provided data that revealed related material and intersecting concerns that linked the opportunity for a shared mitigation; all of these high risk factors are significantly reduced by one common risk control: limiting the duration of travel to within approximately one year.

\section{III.F. The Integrated Solution for Vehicle, Crew, and Mission Risk Reduction}

There is a feasibility and risk gap that exists between current ISS space flight and nearfuture deep-space travel. We propose that the balanced, integrated solution to closing this gap and achieving deep-space travel is to 1) provide modest, reasonable controls for risks associated with an approximately one-year mission, and 2) reduce the transit time to no more than approximately one year. Figure 7 illustrates the existing gap in space flight risk and feasibility with the proposed "sweet spot" of an approximately one-year transit time. ${ }^{3}$

As a result of our conclusion, we further investigated significant reduction of mission duration to Mars, specifically within the confines of currently available chemical-propulsion technology. We assessed only current or readily available technologies to determine, first, if chemical the propulsion capabilities we have to date could produce a feasible mission 


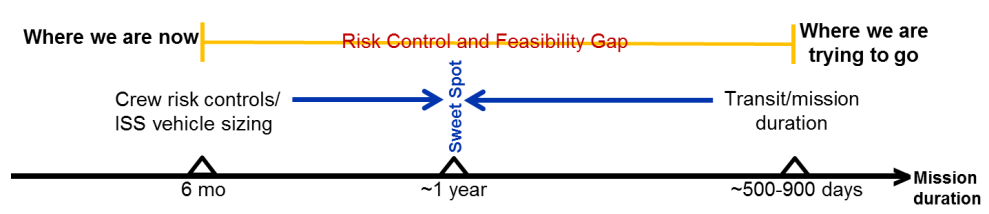

Figure 7. Integrated Solution to Close the Feasibility and Risk Gap ??

solution for a Mars venture occurring as early as the 2030s. It did not seem probable that other propulsion technologies, such as NTP, for example, would be available in sufficient time to support the 2030s time-frame.

\section{III.G. Fast Mars Transfer}

Our review of results in a 2005 paper $^{4}$ from an astrodynamics study at the Goddard Space Flight Center showed possible Mars round trip times of 3-6 months using current chemical propulsion technology. The method utilized in that analysis was based on pre-positioning a four-stage propulsion vehicle in Mars orbit to dock with the incoming crew, allowing them to conduct a short-duration mission, and then returning the crew transit habitat to Earth without waiting for the next synodic period as planned in current Mars design reference missions. In further review of this paper, we noted that the crew transit vehicle mass in the study was modestly sized at $30 \mathrm{t}$, and that there were significantly high $\Delta v$ penalties in the 3 to 6 month trip time. However, the study presented a plausible capability utilizing current or readily available propulsion technologies to pre-position a vehicle in Mars orbit to perform such a mission. We initiated an analysis utilizing this method, but having the following new constraints: determine if any Mars round-trip missions existed between now and 2040 that could be performed within a one year duration, utilizing a vehicle weighing $\sim 50 \mathrm{t}$ ( equivalent to the ISS-Derived Concept weight), with bi-propellant chemical propulsion for the Mars return trip vehicle and the outbound Mars insertion stage, and cryogenic hydrogen for the first three of the four outbound stages leaving Earth.

Our goal was to assess the feasibility of reducing a Mars mission round trip-duration to approximately one year with a crew of four, using current or readily available chemical propulsion capability. In turn, this would serve toward closing the feasibility and risk gap for potential Mars exploration to be realized in the 2030s.

\section{Mission Architecture}

\section{IV.A. Overview}

We employ several key techniques in our study to minimize the Initial Mass in Low Earth Orbit (IMLEO) required by our round-trip trajectories.: pre-positioning, On-Orbit Staging 
(OOS), and specialized round-trip trajectory optimization techniques including the method of embedded trajectory grids and genetic algorithms.

\section{IV.B. Pre-positioning of Mission Elements}

In our study we exploit pre-positioning, in which the propellant and equipment required by the human segment of the mission for operations at Mars (including the propellant for Mars departure and management of Earth atmospheric entry) is transported to Mars via lowerenergy one-way trajectories in advance of the arrival of the astronauts. This mission design yields substantially reduced IMLEO compared to designs without pre-positioning. ${ }^{5}$ The improved performance comes at the cost of increasing the mission complexity and committing the crew to travel to Mars without their Earth-return propellant onboard at the beginning of their journey.

Our constraints for the pre-positioning trajectories are that the pre-position segment of the mission must arrive at Mars no less than 30 days before the Earth-departure date of the astronauts, and the elapsed time between the Earth-departure date of the pre-position mission and the Mars departure date of the humans must not exceed 1095 days ( $~ 3$ years). These constraints are intended to ensure that there is adequate time to verify the integrity of the pre-position payload prior to committing the astronauts to Earth departure, and also to ensure that the total time spent in space by the pre-position system is not so long as to raise concerns about its reliability. Spacecraft systems analysis beyond the scope of our study will be required to refine the numerical values of these pre-position constraints. Also, as with the human segment, we assume that the pre-position mission is assembled in LEO prior to departing Earth, but we do not analyze any aspects of the assembly.

\section{IV.C. On-Orbit Staging (OOS)}

The OOS concept expands upon current technology and designs by applying staging to an assembled structure to achieve higher than currently applied $\Delta v$ levels to decrease interplanetary travel times to less than a year. ${ }^{4}$ We apply the OOS at all appropriate injection and arrival orbital or hyperbolic conditions that are required for transfer to or from planetary bodies. Architecturally, we begin with a set of launch vehicles that place an assembly spacecraft, propulsive elements, and bulk supplies into LEO in advance of mission hardware or crew. All elements are then robotically assembled into several larger elements that permit optimal staging and a significant increase in the ratio of payload mass to initial wet mass. This staging can be thought of as similar to that used for any launch vehicle when traveling from the surface of Earth to LEO, although the elements may not be stacked like traditional Earth-to-orbit vehicles (i.e., one on top of the other). OOS can be applied with any class of 
launch vehicle, with the only measurable difference being the number of launches required to deliver the necessary assets to LEO for a particular mission. The results we present herein assume the use of existing high-performance $\left(\mathrm{LO}_{2} / \mathrm{LH}_{2}\right)$ and storable hypergolic bi-propellant propulsion systems, but comparisons were also performed to include conceptual NTP systems. Additional performance gains can be realized by augmenting the OOS concept with useful infrastructure. A complete treatment of optimal staging can be found in several texts, including Prussing and Conway, ${ }^{15}$ Wiesel,${ }^{16}$ and Curtis. ${ }^{17}$ More detailed discussion of the particular application of OOS used in our study is presented in Ref. 5.

\section{IV.D. Spacecraft Trajectory Design and Optimization}

We consider four types of opposition-class round-trip trajectories to visit Mars in our study: Earth to Mars, Mars to Earth (EMME), Earth to Venus to Mars, Mars to Earth (EVMME), Earth to Mars, Mars to Venus to Earth (EMMVE), and Earth to Venus to Mars, Mars to Venus to Earth (EVMMVE). The EMME trajectories travel directly from Earth to Mars, remain at Mars for some amount of time, and then travel directly from Mars to Earth. The EVMME trajectories perform a flyby of Venus on the way from Earth to Mars and then travel directly from Mars to Earth. The EMMVE trajectories travel directly from Earth to Mars, but then perform a flyby of Venus on the way from Mars to Earth. The EVMMVE trajectories perform a flyby of Venus both on the way from Earth to Mars and on the way from Mars to Earth. In all cases the intent of including Venus flybys is to reduce the total IMLEO for the mission by virtue of utilizing the gravity assist provided by Venus to advantageously manage the spacecraft's velocity without consuming propellant. As detailed in a subsequent section, we found that there are some cases for which including Venus gravity assist offers a small reduction in IMLEO or provides mission deployment opportunities during months/years when there would otherwise be none, but all trajectory sequences involving Venus require the spacecraft to pass within at least Venus's distance from the Sun $(\sim 0.7$ $\mathrm{AU})$. Some of the trajectories that include a Venus flyby pass considerably closer to the Sun (as close as $\sim 0.5 \mathrm{AU}$ ). Even some of the trajectories with no Venus flybys still pass close to the Sun ( 0.55-0.65 AU). We do not recommend any of the cases which pass closer than $0.7 \mathrm{AU}$ to the Sun. Any mission design that requires the spacecraft to pass close to the Sun will naturally involve special spacecraft design considerations for thermal and radiation protection, especially with a human crew aboard. This will clearly impact the spacecraft design (e.g., mass), although the technology readiness levels are currently unclear for thermal and radiation protection systems that will function adequately close to the Sun. In the absence of reliable information for such designs, we elected to not incorporate this into our analysis, and instead simply report closest approach to the Sun for all of our mission trajectories. 
The trajectory analysis software developed for NASA's Near-Earth Object Human Space Flight Accessible Targets Study (NHATS) was modified for the purpose of analyzing EMME trajectories. This software utilizes the method of embedded trajectory grids to perform a comprehensive round-trip trajectory scan across all combinations of Earth departure date, time of flight (TOF) from Earth to Mars, stay time at Mars, and TOF from Mars to Earth, within the constraints specified for our study. This trajectory analysis technique is extensively documented in the context of NHATS in Refs. 18, 19, and 20, and is also described on the NHATS web-site. ${ }^{\text {a }}$ Full precision high-fidelity ephemeris files for Earth and Mars (obtained from the Jet Propulsion Laboratory (JPL) Horizons system, as mentioned previously for pre-position trajectory analysis) are used as inputs to the Lambert solver, which in turn computes the conic heliocentric trajectories of the spacecraft for the Earth-to-Mars and Mars-to-Earth segments of the mission. We employed the method of patched conics to compute the associated $\Delta v$ maneuver magnitudes for Earth departure from a reference circular low-altitude Earth parking orbit, arrival in a highly eccentric Mars parking orbit, and then departure from the highly eccentric Mars parking orbit after the stay time at Mars has elapsed. Earth departure date, TOF from Earth to Mars, stay time at Mars, and TOF from Mars to Earth were all scanned using the embedded trajectory grid software at step sizes of 5 days.

The analysis of the EVMME, EMMVE, and EVMMVE cases was performed using a variant of the Evolutionary Mission Trajectory Generator (EMTG). EMTG is a global optimization tool intended for the design of high-thrust and low-thrust trajectories with or without planetary flybys. ${ }^{21,22,23}$ We created a variant of EMTG especially suited to the analysis performed for this study. EMTG contains several different trajectory models suitable for different types of missions. The model that we used in this work is the Multiple Gravity Assist (MGA) model, first described by Vinko and Izzo $^{24}$ with some simplifying assumptions. First, all rocket burns are assumed to be impulsive. Second, impulses are allowed only at launch and at the moment of periapse passage in each gravity-assist maneuver. Finally, a twobody, patched-conic model is used, allowing the path of the spacecraft between two bodies to be modeled using the Lambert solution to the problem of finding the conic section orbit between two points separated by a specified amount of time. ${ }^{15}$ The specific Lambert solver used was developed by Izzo et al. for the Global Trajectory Optimization Problem (GTOP) database. ${ }^{25}$ The resulting Lambert arcs are patched at each intermediate destination. Since both Lambert arcs are guaranteed to touch one endpoint to the position of the planet, we must only account for the difference in velocity between the end point of the incoming arc and the starting point of the outgoing arc. This velocity discrepancy is resolved by modeling the flyby about the planet.

\footnotetext{
${ }^{a}$ http://neo.jpl.nasa.gov/nhats/, accessed on 2013-07-02.
} 
Our objective was to investigate trades between Earth-departure date, flight time, and required IMLEO. These trades were evaluated using a combination of a grid search and a Multi-Objective Genetic Algorithm (MOGA), specifically Non-Dominated Sorting Genetic Algorithm II (NSGA-II). ${ }^{26}$ NSGA-II finds the Pareto-optimal set of non-dominated solutions to a problem with two or more objective functions; i.e., the set of solutions where no solution is superior to any other in all of the objective metrics. In this case, NSGA-II is used to find the trade curve between total mission duration and IMLEO for round-trip Mars trajectories given a fixed Earth-departure date. NSGA-II varies the time spent at Mars and the phase flight time, and for each candidate solution EMTG computes the $\Delta v$ magnitudes at departure from and arrival at both Earth and Mars as well as as the $\Delta v$ at the periapse of each flyby. The total mission duration is then computed as the sum of all phase flight times and the time spent at Mars, and the IMLEO is computed using the OOS algorithm described in a previous section. NSGA-II was used to trade mission duration versus IMLEO for a set of fixed Earth-departure dates. Each run of NSGA-II was used to create a scan line on a Pork Chop Contour (PCC) plot, with mission duration on the ordinate, Earth-departure date on the abscissa, and IMLEO (or equivalent number of launch vehicles) represented by color. This data set was then mined to find the best launch opportunities for each year from 2018 to 2040 .

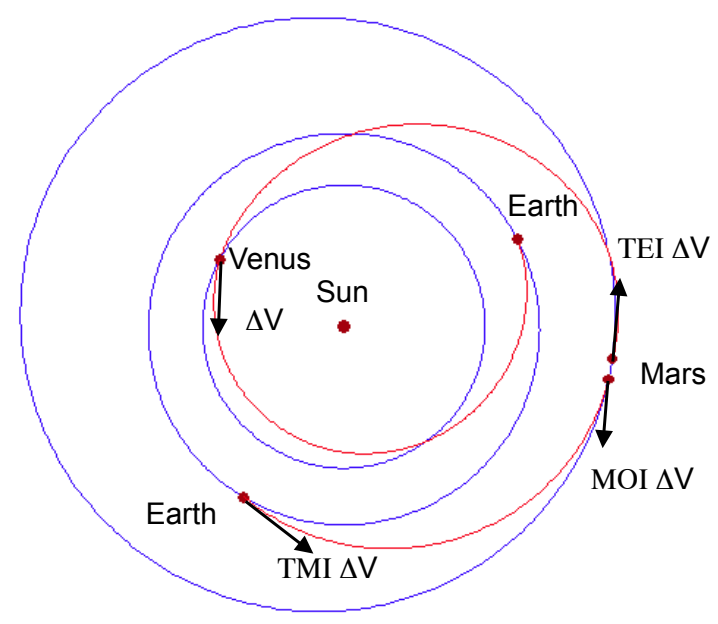

(a) (a)

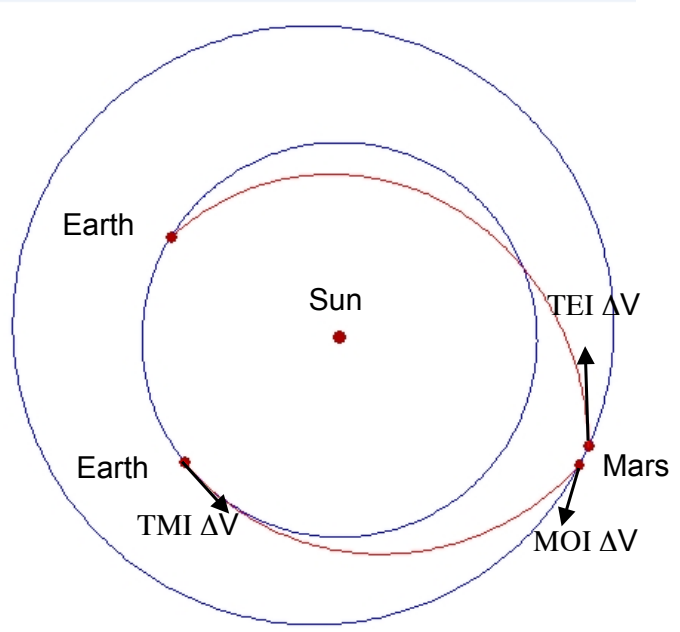

(b) (b)

Figure 8. (a) Optimal one year duration round-trip trajectory to Mars with a Venus gravity assist on the Earth-return portion of the trajectory, (b) Optimal one year duration round-trip trajectory to Mars without a Venus gravity assist 


\section{IV.E. Lean Fast Mars Transfer Architecture Development}

In September 2012, we used Goddard Space Flight Center (GSFC)'s Architecture Design Laboratory (ADL) to develop a systems architecture to assess the mission described by the flight dynamics team. The principal members of the ADL team included the flight dynamics team, propulsion engineering, mechanical systems, and mission systems engineering. The flight dynamics concept was used as the basis of priority mission requirements, as well as the mission-critical elements to support the crew on their journey to Mars and back, as well as visiting the surface. The ADL team developed a design that described propellant tank sizes, engine types, and mechanical structure for each phase of the Fast Mars Transfer, as well as a conceptual method for parallel staging to maximize the mass of the payload for each phase.

In order to develop a feasible architecture for the Lean Fast Mars Transfer concept, the ADL utilized traditional systems engineering processes to break the work into an orderly flow. This enabled a systems-oriented process to define and, subsequently, provide design solutions for Lean Fast Mars Transfer. In summary, our ADL timeline consisted of:

1. Development of end-to-end mission requirements, including customer-supplied payloads and their requirements to fulfill the mission

2. Development of a concept of operations (conops) that fulfills all requirements for the Lean Fast Mars Transfer mission

3. Detailed analysis of the propulsion requirements

4. Definition of all required propulsion components

5. Development of a mechanical concept that allows launching on the minimum number of SLS launch vehicles, on-orbit assembly, on-orbit staging, and supplying all required $\Delta v$ in support of the mission conops

\section{IV.F. Summary of Mission Requirements and Concept of Operations}

In order to successfully send a crew to Mars, have them land, and then return them to Earth within a period of one year, the ADL was given the following top-level requirements, and developed a concept of operations:

1. Phase 1, Pre-position in Mars orbit via a low energy transfer an Earth Return Reentry Vehicle (ERRV), A Descent/Ascent Vehicle (D/AV), and a MPLM, along with the propulsion unit required to return the crew to earth (Trans-Earth Injection (TEI) stage) 
(a) Phase 1 Trans-Mars Injection (TMI) and Mar Orbit Injection (MOI) stages were developed by the ADL

2. Phase 2, Launch the crew to Mars on a high-energy, fast trajectory, utilizing a DSH, MPLM, and Pressurized Cargo Module (PCM)

(a) The MPLM and PCM are decoupled from the stack prior to MOI to reduce $\Delta v$ requirements.

(b) Phase 2 TMI and MOI stages were developed by the ADL

3. Phase 3, Phase 1 and 2 elements rendezvous and dock in Mars orbit. Crew utilized $\mathrm{D} / \mathrm{AV}$ to land on the Mars surface and return to the integrated stack. After landing, the $\mathrm{D} / \mathrm{AV}$ is decoupled from the stack

4. Phase 4, The crew is returned to Earth on a high-energy, fast trajectory, utilizing an MPLM, DSH, and ERRV

(a) A TEI stage was developed by the ADL.

(b) The ERRV decouples prior to atmospheric re-entry, utilizing the Earth's atmosphere to safely return.

The supplied equipment is detailed in Figure 9.

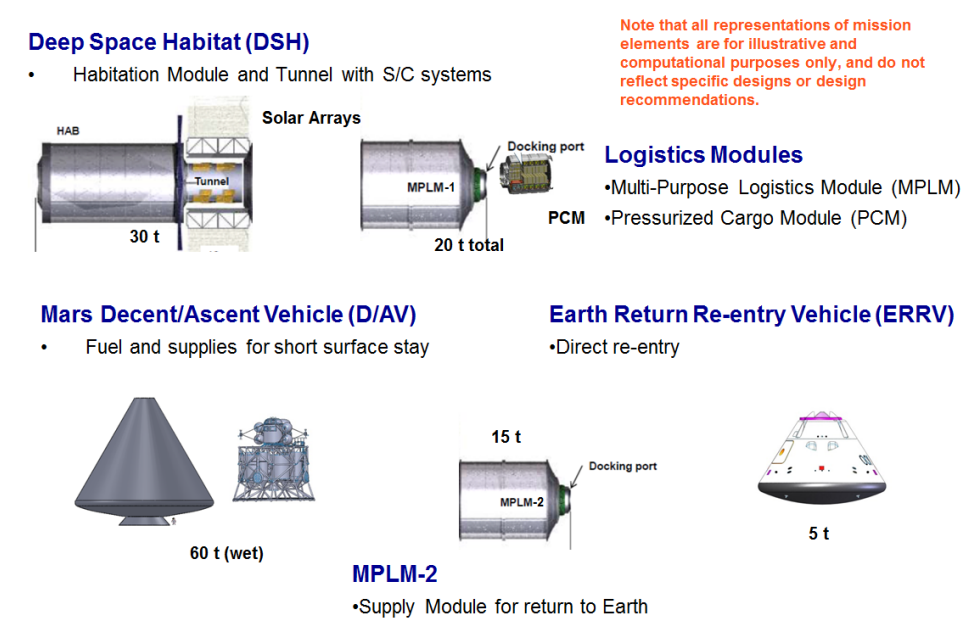

Figure 9. Required Crew Equipment for Lean Fast Mars Transfer

For the mission design, a cryogenic liquid hydrogen/oxygen system was chosen for the design of all TMI stages. Recent technological advances have resulted in essentially zeroboiloff systems for upwards of six months as shown in Figure 10. Hypergolic chemical propulsion was selected for MOI and TEI stages, due to extended hold-time requirements. 


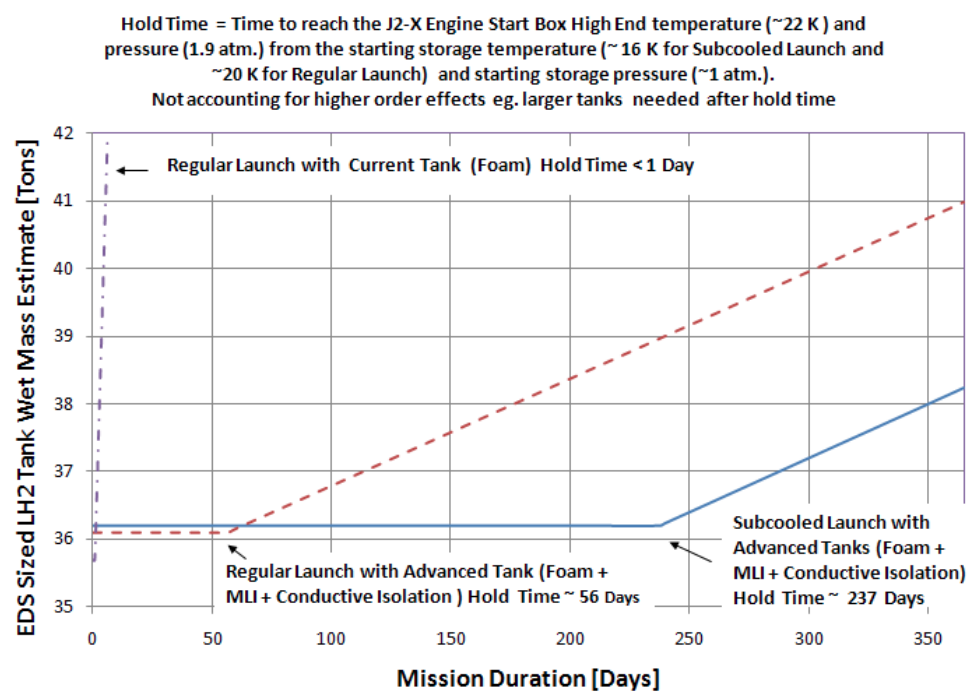

Figure 10. EDS size $\mathrm{H}_{2}$ tank wet mass vs mission duration - a preliminary estimate

For sub-cooled cryogenic storage, a mass fraction of $3.2 \%$ of the stored cryogen for the $6+$ month zero-boiloff case was calculated. The ADL assumed an additional $0.8 \%$ for structural mass and conservativeness. These mechanical mass fractions are conservative, as demonstrated by the mechanical mass fractions for large ground-launched systems, such as the Space Transportation System (STS) External Tank, which had to endure significantly higher g loads as well as aerodynamic loads.

\section{IV.G. Description of ADL-developed stages for each phase of the Lean Fast Mars Transfer concept}

The Phase 1 elements that are used to pre-position assets in Mars orbit are shown in Figure 11.

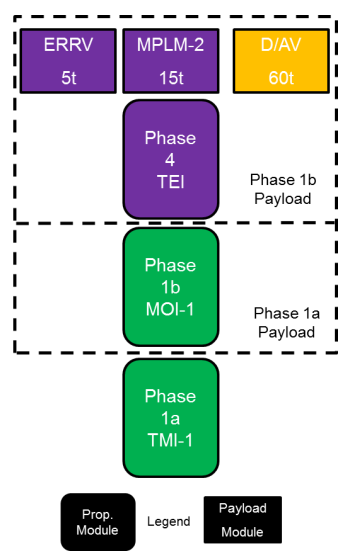

Figure 11. Block diagram of Phase 1 elements

Figure 12 shows the design details for the phase 1 TMI and MOI stages. Tables 1 and 
2 describe the propulsion and propellant loading for each stage in Phase 1a and Phase 1b, respectively. Table 3 describes the distribution of hardware among the eight launches that make up Phase 1. Note that the full mass capacity of SLS 4-1 is not used because the payload of SLS 4-1 uses all of the available volume despite being low in mass.

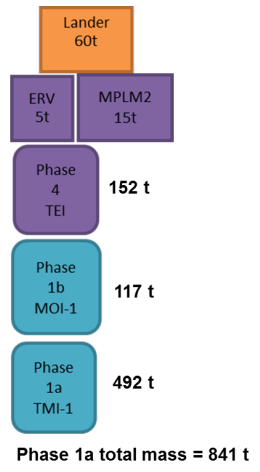

(a)

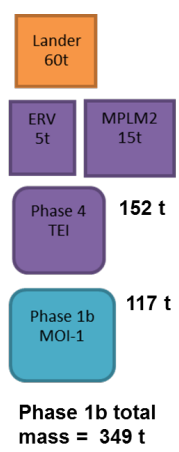

(b)

Figure 12. Mass Breakdown of (a) Phase 1a TMI Elements and (b) Phase 1b MOI Elements

\begin{tabular}{llllllll}
\hline \hline Stage & $\begin{array}{l}\text { Isp } \\
(\mathrm{s})\end{array}$ & $\begin{array}{l}\text { Propellant } \\
(\mathrm{kg})\end{array}$ & Engine & $\begin{array}{l}\text { Thrust } \\
(\mathrm{kN})\end{array}$ & \# of Engines & $\begin{array}{l}\text { Total Thrust } \\
(\mathrm{kN})\end{array}$ & $\begin{array}{l}\text { Burn Time } \\
(\mathrm{s})\end{array}$ \\
\hline 1 & 465 & 114500 & J-2XX +3 RL60 & 1870 & Set & 2150 & 243 \\
2 & 465 & 114500 & RL-60 & 280 & 3 & 840 & 622 \\
3 & 465 & 114500 & RL-60 & 280 & 3 & 560 & 622 \\
4 & 465 & 114500 & RL-60 & 280 & 3 & 560 & 622 \\
\hline Total & 458000 & & & & & 2108 \\
\hline \hline
\end{tabular}

Table 1. Stage propulsion and propellant loads for Phase 1a

\begin{tabular}{llllllll}
\hline \hline Stage & $\begin{array}{l}\text { Isp } \\
(\mathrm{s})\end{array}$ & $\begin{array}{l}\text { Propellant } \\
(\mathrm{kg})\end{array}$ & Engine & $\begin{array}{l}\text { Thrust } \\
(\mathrm{kN})\end{array}$ & \# of Engines & $\begin{array}{l}\text { Total Thrust } \\
(\mathrm{kN})\end{array}$ & $\begin{array}{l}\text { Burn Time } \\
(\mathrm{s})\end{array}$ \\
\hline 1 & 338.8 & 27101 & Boeing RS-72 & 53 & 4 & 212 & 425 \\
2 & 338.8 & 27101 & Boeing RS-72 & 53 & 4 & 212 & 425 \\
3 & 338.8 & 27101 & Boeing RS-72 & 53 & 4 & 212 & 425 \\
4 & 338.8 & 27101 & Boeing RS-72 & 53 & 4 & 212 & 425 \\
\hline Total & 108404 & & & & 1699 \\
\hline \hline
\end{tabular}

Table 2. Stage propulsion and propellant loads for Phase 1b 


\begin{tabular}{ccc}
\hline \hline Flight element & Mass (metric tons) & Equipment aboard \\
\hline SLS 1-1 & 117 & Structure, MOI stages \\
SLS 1-2 & 117 & TEI structure and stages \\
SLS 1-3 & 117 & TEI structure and stages \\
SLS 1-4 & 78 & MPLM2, ERRV, D/AV \\
SLS 1-5 & 119 & Cryogenic TMI stages and engine \\
SLS 1-6 & 119 & Cryogenic TMI stages and engine \\
SLS 1-7 & 119 & Cryogenic TMI stages and engine \\
SLS 1-8 & 119 & Cryogenic TMI stages and engine \\
\hline \hline
\end{tabular}

Table 3. Launch vehicle manifest for Phase 1, delivered to LEO for assembly

Figure 13 is an isometric view, showing the entire phase 1 stack, with TMI, MOI, TEI, and required crew equipment.

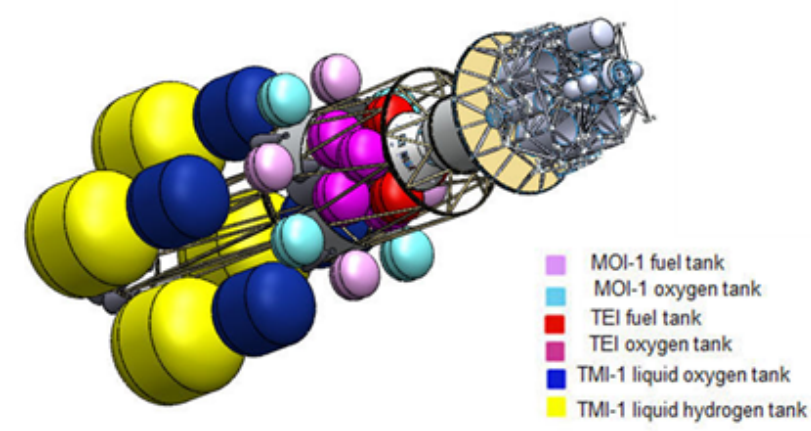

Figure 13. Isometric view of Phase 1 elements

Phase 2 equipment, consisting of an MPLM, PCM, TMI stage, and MOI stage, is shown in Figure 14. The Phase 2 stack conveys the crew to Mars orbit.

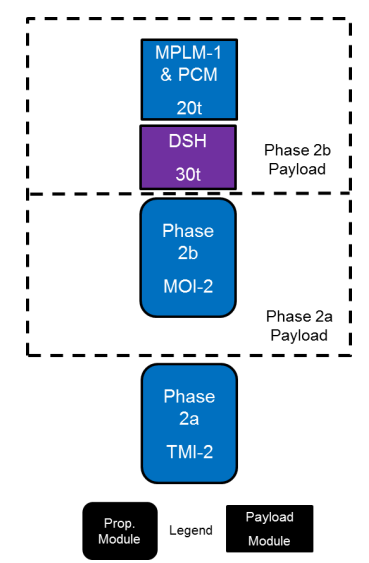

Figure 14. Block diagram of Phase 2 elements 
Figure 15 shows the design details for the phase 2 TMI and MOI stages. Tables 4 and 5 describe the propulsion and propellant loading for each stage in Phase $2 \mathrm{a}$ and Phase 2b, respectively. Table 6 describes the distribution of hardware among the six launches that make up Phase 2. Note that two of the launches are relatively low mass, 72 and 53 tons respectively. The payloads of SLS 2-1 and SLS 2-2 completely fill the launch vehicle fairing even though the full mass capability is not used, leaving no room for additional payload.

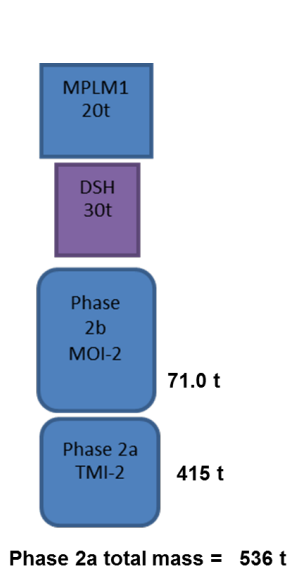

(a)

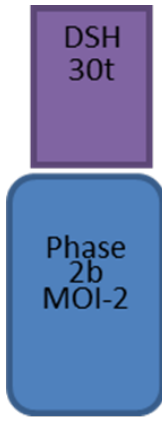

Phase 2b

total mass

$=101 \mathrm{t}$
$71.0 \mathrm{t}$

(b)

Figure 15. Mass Breakdown of (a) Phase 2a TMI Elements and (b) Phase 2b MOI Elements

\begin{tabular}{|c|c|c|c|c|c|c|c|}
\hline Stage & $\begin{array}{l}\text { Isp } \\
(\mathrm{s})\end{array}$ & $\begin{array}{l}\text { Propellant } \\
(\mathrm{kg})\end{array}$ & Engine & $\begin{array}{l}\text { Thrust } \\
(\mathrm{kN})\end{array}$ & \# of Engines & $\begin{array}{l}\text { Total Thrust } \\
(\mathrm{kN})\end{array}$ & $\begin{array}{l}\text { Burn Time } \\
\text { (s) }\end{array}$ \\
\hline 1 & 465 & 96563 & $\begin{array}{l}\text { J-2XX } \\
+2 \text { RL-60 }\end{array}$ & 1870 & Set & 1870 & 235.5 \\
\hline 2 & 465 & 96563 & RL-60 & 280 & 2 & 560 & 786.3 \\
\hline 3 & 465 & 96563 & RL-60 & 280 & 2 & 560 & 786.3 \\
\hline 4 & 465 & 96563 & RL-60 & 280 & 2 & 560 & 786.3 \\
\hline Total & & 386252 & & & & & 2594 \\
\hline
\end{tabular}

Table 4. Stage propulsion and propellant loads for Phase 2a 


\begin{tabular}{llllllll}
\hline \hline Stage & $\begin{array}{l}\text { Isp } \\
(\mathrm{s})\end{array}$ & $\begin{array}{l}\text { Propellant } \\
(\mathrm{kg})\end{array}$ & Engine & $\begin{array}{l}\text { Thrust } \\
(\mathrm{kN})\end{array}$ & \# of Engines & $\begin{array}{l}\text { Total Thrust } \\
(\mathrm{kN})\end{array}$ & $\begin{array}{l}\text { Burn Time } \\
(\mathrm{s})\end{array}$ \\
\hline 1 & 338.8 & 16519 & Boeing RS-72 & 53 & 4 & 212 & 258.9 \\
2 & 338.8 & 16519 & Boeing RS-72 & 53 & 4 & 212 & 258.9 \\
3 & 338.8 & 16519 & Boeing RS-72 & 53 & 2 & 106 & 517.8 \\
4 & 338.8 & 16519 & Boeing RS-72 & 53 & 2 & 106 & 517.8 \\
\hline Total & 66076 & & & & 1553 \\
\hline \hline
\end{tabular}

Table 5. Stage propulsion and propellant loads for Phase $2 \mathrm{~b}$

\begin{tabular}{ccc}
\hline \hline Flight element & Mass (metric tons) & Equipment aboard \\
\hline SLS 2-1 & 72 & Structure, MOI stages \\
SLS 2-2 & 53 & DSH, MPLM1, PCM \\
SLS 2-3 & 101 & Cryogenic TMI stages and engine \\
SLS 2-4 & 101 & Cryogenic TMI stages and engine \\
SLS 2-5 & 101 & Cryogenic TMI stages and engine \\
SLS 2-6 & 101 & Cryogenic TMI stages and engine \\
\hline \hline
\end{tabular}

Table 6. Launch vehicle manifest for Phase 2, delivered to LEO for assembly. Does not include crew.

Figure 16 is an isometric view, showing the entire phase 2 stack, with TMI, MOI, and required crew equipment.

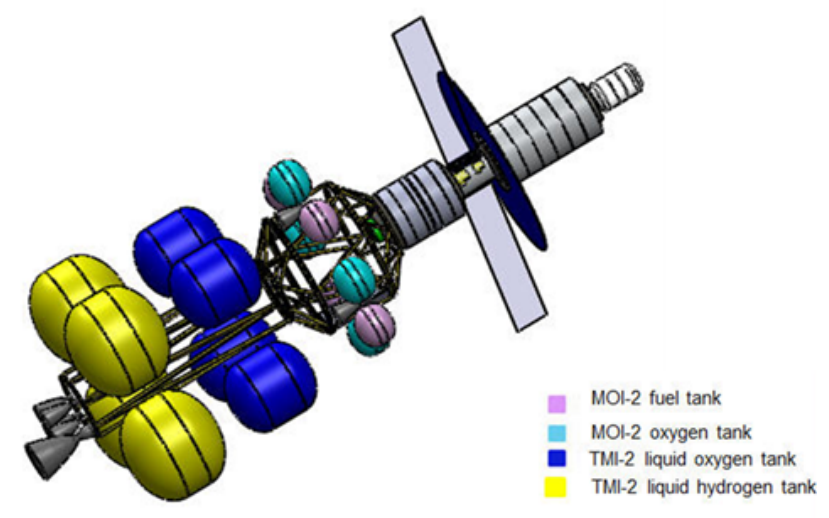

Figure 16. Isometric view of Phase 2 elements

Phase 3 consists of Mars orbit rendezvous and descent/ascent. The vehicle elements involved in Phase 3 are shown in Figure 17. 

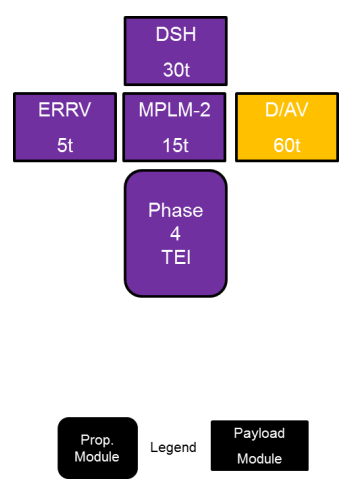

Figure 17. Block diagram of Phase 3 elements

Phase 4 is the return of the crew to Earth, utilizing the pre-positioned MPLM, the DSH, and the ERRV. The TEI stage propels the crewed equipment to Earth on a high-energy trajectory. The TEI stage, MPLM, and DSH are jettisoned prior to Earth re-entry. The ERRV safely conveys the crew back to Earth through atmospheric re-entry. Figures 18 and 19 show the design details of the Phase 4 equipment in block diagram form, Table 7 outlines the propulsion systems and propellant loading of the Phase 4 stages, and Figure 20 is an isometric representation of the Phase 4 vehicle.

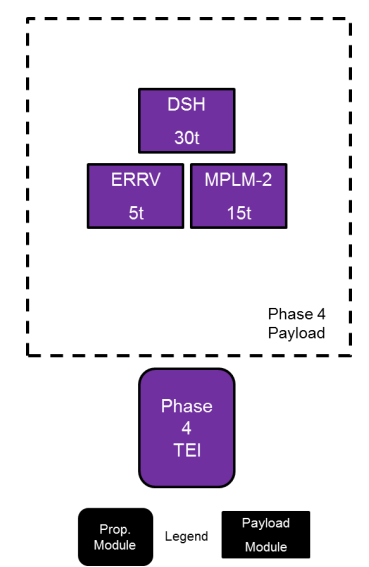

Figure 18. Block diagram of Phase 4 elements

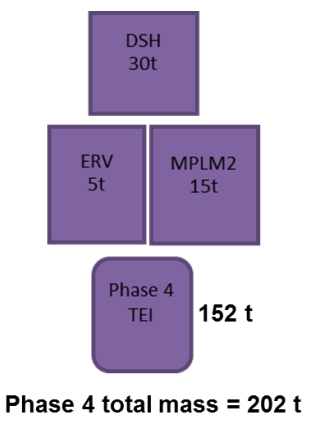

Figure 19. Mass Breakdown of Phase 4 Elements 


\begin{tabular}{|c|c|c|c|c|c|c|c|}
\hline Stage & $\begin{array}{l}\text { Isp } \\
(\mathrm{s})\end{array}$ & $\begin{array}{l}\text { Propellant } \\
(\mathrm{kg})\end{array}$ & Engine & $\begin{array}{l}\text { Thrust } \\
(\mathrm{kN})\end{array}$ & \# of Engines & $\begin{array}{l}\text { Total Thrust } \\
(\mathrm{kN})\end{array}$ & $\begin{array}{l}\text { Burn Time } \\
\text { (s) }\end{array}$ \\
\hline \multirow[t]{2}{*}{1} & 338.8 & 35406 & 1 LR-91-11 & 573 & Set & 573 & 1109.8 \\
\hline & & & +2 Boeing RS-72 & & & & \\
\hline 2 & 338.8 & 35406 & Boeing RS-72 & 53 & 2 & 106 & 1109.8 \\
\hline 3 & 338.8 & 35406 & Boeing RS-72 & 53 & 2 & 106 & 1109.8 \\
\hline 4 & 338.8 & 35406 & Boeing RS-72 & 53 & 2 & 106 & 205.3 \\
\hline Total & & 141624 & & & & & 3535 \\
\hline
\end{tabular}

Table 7. Stage propulsion and propellant loads for Phase 4

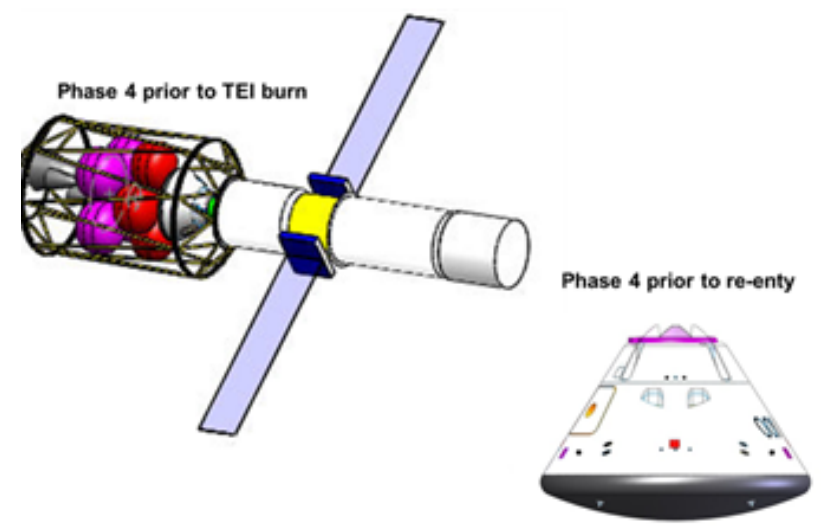

Figure 20. Isometric view of Phase 4 elements

\section{Discussion}

\section{V.A. Discussion of Trajectory Analysis}

Figure 21 shows the annual minimum number of launches for each year between 2018 and 2040, inclusive. Results are shown for Cases 3 ("Cryo \& Bi-prop" in red), 5 ("Cryo Only" in green), and 9 ("NTP Only" in blue). A black line across Figure 21 indicates 20 heavy-lift (120 t to LEO) launches. Case 3 only yields solutions requiring less than 20 launches during the years 2019, 2020, and 2034. Case 5 yields $<20$ launch solutions during the years 2018, 2019, 2020, 2022, and 2033-2037, inclusive. Case 9 yields solutions requiring substantially less than 20 launches during each year considered except 2023 and 2040. No solutions are available during the years 2023 and 2040 for total mission $\Delta v \leq 30 \mathrm{~km} / \mathrm{s}$ because Earth and Mars are on the same side of the Sun, with Earth ahead of Mars. Hence any trajectories from Earth to Mars would have to be retrograde relative to Earth's heliocentric orbit. 


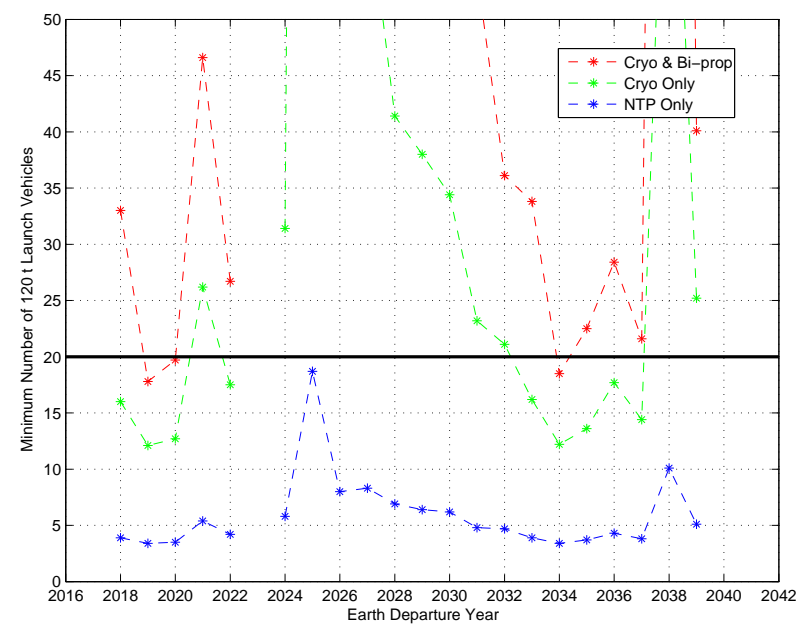

Figure 21. Annual Minimum Number of Launches for Cases 3, 5, and 9 (EMME) With a 100 t Human Spacecraft Dry Mass

Table 8. Annual Minimum Total \# Launches Mission Solutions For Cases 3, 5, and 9 (EMME) With a 100 t Human Spacecraft Dry Mass

\begin{tabular}{c|ccc|ccc|ccc}
\hline & \multicolumn{3}{|c|}{ Case 3 } & \multicolumn{3}{c|}{ Case 5 } & \multicolumn{3}{c}{ Case 9 } \\
\hline $\begin{array}{c}\text { Earth } \\
\text { Dep. }\end{array}$ & $\begin{array}{c}\text { Total } \\
\text { \# LV }\end{array}$ & $\begin{array}{c}\text { Total } \\
\text { Duration } \\
\text { (days) }\end{array}$ & $\begin{array}{c}\text { Closest } \\
\text { Approach } \\
\text { to Sun (AU) }\end{array}$ & $\begin{array}{c}\text { Total } \\
\text { \# LV }\end{array}$ & $\begin{array}{c}\text { Total } \\
\text { Duration } \\
\text { (days) }\end{array}$ & $\begin{array}{c}\text { Closest } \\
\text { Approach } \\
\text { to Sun (AU) }\end{array}$ & $\begin{array}{c}\text { Total } \\
\text { \# LV }\end{array}$ & $\begin{array}{c}\text { Total } \\
\text { Duration } \\
\text { (days) }\end{array}$ & $\begin{array}{c}\text { Closest } \\
\text { Approach } \\
\text { to Sun (AU) }\end{array}$ \\
\hline 2018 & 33.0 & 370 & 0.77 & 16.0 & 370 & 0.76 & 3.9 & 370 & 0.76 \\
2019 & 17.8 & 362 & 0.73 & 12.1 & 362 & 0.73 & 3.4 & 362 & 0.73 \\
2020 & 19.7 & 362 & 0.77 & 12.7 & 362 & 0.77 & 3.5 & 362 & 0.77 \\
2021 & 46.6 & 370 & 0.64 & 26.2 & 370 & 0.64 & 5.4 & 370 & 0.64 \\
2022 & 26.7 & 362 & 0.71 & 17.5 & 362 & 0.71 & 4.2 & 362 & 0.71 \\
2023 & - & - & - & - & - & - & - & - & - \\
2024 & 51.8 & 362 & 0.66 & 31.4 & 362 & 0.66 & 5.8 & 362 & 0.66 \\
2025 & 3038.1 & 370 & 0.44 & 360.6 & 370 & 0.44 & 18.7 & 370 & 0.44 \\
2026 & 149.5 & 370 & 0.69 & 55.8 & 370 & 0.61 & 8.0 & 370 & 0.60 \\
2027 & 194.9 & 365 & 0.57 & 61.2 & 370 & 0.56 & 8.3 & 370 & 0.56 \\
2028 & 115.2 & 370 & 0.68 & 41.4 & 370 & 0.68 & 6.9 & 370 & 0.68 \\
2029 & 104.6 & 370 & 0.63 & 38.0 & 370 & 0.63 & 6.4 & 370 & 0.63 \\
2030 & 80.2 & 362 & 0.63 & 34.4 & 370 & 0.78 & 6.2 & 370 & 0.78 \\
2031 & 54.1 & 370 & 0.68 & 23.2 & 370 & 0.68 & 4.8 & 370 & 0.67 \\
2032 & 36.1 & 362 & 0.70 & 21.1 & 362 & 0.72 & 4.7 & 362 & 0.70 \\
2033 & 33.8 & 370 & 0.74 & 16.2 & 370 & 0.75 & 3.9 & 370 & 0.74 \\
2034 & 18.5 & 362 & 0.73 & 12.2 & 362 & 0.74 & 3.4 & 362 & 0.74 \\
2035 & 22.5 & 362 & 0.81 & 13.6 & 362 & 0.81 & 3.7 & 362 & 0.81 \\
2036 & 28.4 & 370 & 0.69 & 17.7 & 370 & 0.69 & 4.3 & 370 & 0.69 \\
2037 & 21.6 & 362 & 0.73 & 14.4 & 362 & 0.73 & 3.8 & 362 & 0.73 \\
2038 & 238.2 & 370 & 0.56 & 82.4 & 370 & 0.56 & 10.1 & 370 & 0.56 \\
2039 & 40.1 & 362 & 0.68 & 25.2 & 362 & 0.68 & 5.1 & 362 & 0.68 \\
2040 & - & - & - & - & - & - & - & - & - \\
& & & & & & & & & \\
\end{tabular}

\section{V.B. Discussion of Our Point Design}

The results from GSFC's ADL study indicate that volumetric constraints of the SLS system will drive several more SLS unnecessary launches than if we could manifest each vehicle with 
Table 9. Annual Minimum Total \# Launches Mission Solutions For Cases 3, 5, and 9 (EMME) With a 50 t Human Spacecraft Dry Mass

\begin{tabular}{c|ccc|ccc|ccc}
\hline & \multicolumn{3}{|c|}{ Case 3 } & \multicolumn{3}{c|}{ Case 5 } & \multicolumn{3}{c}{ Case 9 } \\
\hline $\begin{array}{c}\text { Earth } \\
\text { Dep. }\end{array}$ & $\begin{array}{c}\text { Total } \\
\text { \# LV }\end{array}$ & $\begin{array}{c}\text { Total } \\
\text { Duration } \\
\text { (days) }\end{array}$ & $\begin{array}{c}\text { Closest } \\
\text { Approach } \\
\text { to Sun (AU) }\end{array}$ & $\begin{array}{c}\text { Total } \\
\text { \# LV }\end{array}$ & $\begin{array}{c}\text { Total } \\
\text { Duration } \\
\text { (days) }\end{array}$ & $\begin{array}{c}\text { Closest } \\
\text { Approach } \\
\text { to Sun (AU) }\end{array}$ & $\begin{array}{c}\text { Total } \\
\text { \# LV }\end{array}$ & $\begin{array}{c}\text { Total } \\
\text { Duration } \\
\text { (days) }\end{array}$ & $\begin{array}{c}\text { Closest } \\
\text { Approach } \\
\text { to Sun (AU) }\end{array}$ \\
\hline 2018 & 16.5 & 370 & 0.77 & 8.0 & 370 & 0.76 & 1.9 & 370 & 0.76 \\
2019 & 8.9 & 362 & 0.73 & 6.0 & 362 & 0.73 & 1.7 & 362 & 0.73 \\
2020 & 9.9 & 362 & 0.77 & 6.3 & 362 & 0.77 & 1.8 & 362 & 0.77 \\
2021 & 23.3 & 370 & 0.64 & 13.1 & 370 & 0.64 & 2.7 & 370 & 0.64 \\
2022 & 13.4 & 362 & 0.71 & 8.8 & 362 & 0.71 & 2.1 & 362 & 0.71 \\
2023 & - & - & - & - & - & - & - & - & - \\
2024 & 25.9 & 362 & 0.66 & 15.7 & 362 & 0.66 & 2.9 & 362 & 0.66 \\
2025 & 1519.0 & 370 & 0.44 & 180.3 & 370 & 0.44 & 9.3 & 370 & 0.44 \\
2026 & 74.7 & 370 & 0.69 & 27.9 & 370 & 0.61 & 4.0 & 370 & 0.60 \\
2027 & 97.4 & 365 & 0.57 & 30.6 & 370 & 0.56 & 4.2 & 370 & 0.56 \\
2028 & 57.6 & 370 & 0.68 & 20.7 & 370 & 0.68 & 3.4 & 370 & 0.68 \\
2029 & 52.3 & 370 & 0.63 & 19.0 & 370 & 0.63 & 3.2 & 370 & 0.63 \\
2030 & 40.1 & 362 & 0.63 & 17.2 & 370 & 0.78 & 3.1 & 370 & 0.78 \\
2031 & 27.1 & 370 & 0.68 & 11.6 & 370 & 0.68 & 2.4 & 370 & 0.67 \\
2032 & 18.1 & 362 & 0.70 & 10.6 & 362 & 0.72 & 2.3 & 362 & 0.70 \\
2033 & 16.9 & 370 & 0.74 & 8.1 & 370 & 0.75 & 2.0 & 370 & 0.74 \\
2034 & 9.3 & 362 & 0.73 & 6.1 & 362 & 0.74 & 1.7 & 362 & 0.74 \\
2035 & 11.3 & 362 & 0.81 & 6.8 & 362 & 0.81 & 1.8 & 362 & 0.81 \\
2036 & 14.2 & 370 & 0.69 & 8.8 & 370 & 0.69 & 2.1 & 370 & 0.69 \\
2037 & 10.8 & 362 & 0.73 & 7.2 & 362 & 0.73 & 1.9 & 362 & 0.73 \\
2038 & 119.1 & 370 & 0.56 & 41.2 & 370 & 0.56 & 5.1 & 370 & 0.56 \\
2039 & 20.0 & 362 & 0.68 & 12.6 & 362 & 0.68 & 2.6 & 362 & 0.68 \\
2040 & - & - & - & - & - & - & - & - & - \\
& & & & & & & & & \\
\end{tabular}

its launch capacity. Even with these inefficiencies, the architecture developed by the ADL shows that a short-duration crewed mission to Mars is feasible utilizing existing technology and techniques. The on-orbit storage of cryogenic propulsion is currently being developed for utilization on interplanetary probes, and would enable a significantly more efficient crewed Mars mission. In addition, if NTP were to be developed, this technology would be essential. The mass fraction of the overall system was conservatively estimated. A further engineering study to better refine and validate the concept of operations would be very valuable.

Figure 22 shows the charting of data from Table 9, illustrating the number of launch vehicles required for a 12-month round trip to Mars for a crew transit vehicle mass of $50 \mathrm{t}$, which we selected to be deliberately in family with the ISS-Derived Concept vehicle mass of $45.5 \mathrm{t}$ for a crew of four. Case 3 is represented by the red curve, Case 5 is represented by the green curve, and Case 9 is represented by the blue curve. We have labeled two separate groupings, each identified with a yellow circle, which show launch opportunities occurring during years that match the minimal distance between Earth and Mars according to an approximately 15-year cycle. We noted that this data shows remarkably modest scatter among the three different propulsive method curves for the years identified that are encircled 
in yellow. The results that were computed utilizing current chemical propulsion technology, represented by the red line, show that an average of about 10 launches total are required to launch and preposition all necessary assets for round trips to Mars occurring during the years of 2034, 2035, and 2037 for a crew of four having a 50 t transit vehicle. We advocate that this second launch opportunity grouping, that occurs in the mid-2030s, is much more tenable than to pursue such an undertaking as early as the years of 2019 or 2020. Therefore, we recommend focus on the launch opportunities presented in the mid-2030s.

A sensitivity study ${ }^{27}$ was also conducted which showed that for every one ton of crew transit vehicle mass, there are approximately 26 tons of propellant mass added to the total launch mass. This of course also implies that there is relief in the number of launches to below the 10 launch total if further analysis were to be repeated with a reduction in crew size to perhaps two or three crew-members instead of four, and the vehicle mass would then be reduced in a commensurate manner. As such, we would expect that if for example the vehicle mass were reduced to $30 \mathrm{t}$ to accommodate a crew of two, then this would reduce the number of launches to 6 (20 t less x $26=520 \mathrm{t}$ of propellant reduction, which is the equivalent to reductions of well over four $120 \mathrm{t}$ SLSs). There are other analyses that can also be performed to reduce number of launches, to include stretching the round trip time from 12 months to perhaps 13 or 14 months, which would help reduce the amount of propellant necessary without creating a significant change to the fundamental transit vehicle design, risk controls, or weight. These are some examples of forward analysis work we would hope to explore in order to reduce the number of launches, among other studies of interest.

Figure 22 includes some other significant conclusions of particular interest, however. We have added an overlay indicating the timetable of the 11-year solar cycle, which notably shows that the launch opportunities encircled in the mid-2030s are serendipitously occurring during a solar maximum period. We observed that this coincidental opportunity, having the solar cycle maximum occurring at the same time as a minimal distance between the Earth and Mars, is not a regular phenomenon and in fact will not occur again for a few decades. Therefore, it is a noteworthy and welcome occurrence that our favored launch opportunities (in the mid-2030s) are aligning during a solar maximum period, since solar maximum conditions can potentially provide significant reduction of GCR exposure as previously discussed (as much as half). As such, we would submit that this successfully meets the intended objective of minimizing crew and vehicle risk with regard to GCR, both by reducing the transit duration time, as well as by traveling during a period of solar maximum.

We propose options for executing a lean fast Mars mission, to include either:

1. a brief manned orbit (approximately 1-2 weeks) to possibly conduct telerobotic operations of a prepositioned rover, or deploy a robotic sample probe from off of the crew vehicle onto the surface, or perhaps perform an orbital-capture of a probe previously 


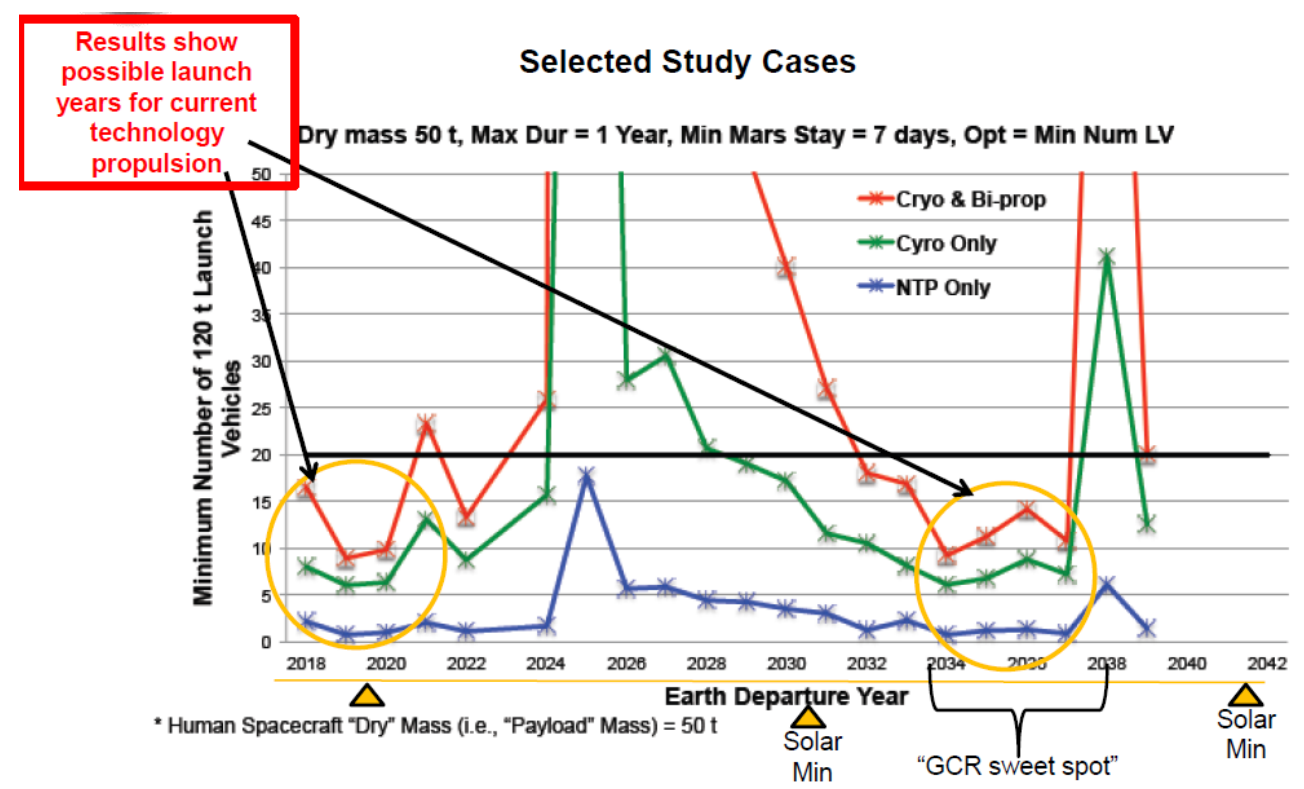

Figure 22. Possible Launch Opportunities Identified in the 2030s for a 12-month Round-Trip to Mars for a 50 t Crew Transit Vehicle

sent to the martian surface that contains a sample which is launched to orbit to dock with the crew vehicle

2. a brief manned landing (approximately 1 week) to perform various sample collections and/or simple on-site evaluations and photo-documentation, and plant flag.

We would also propose to first perform an unmanned end-to-end check out of a full-scale or partial scale mission that verifies performance of the critical mission elements to include remote automated rendezvous and docking of the propulsion stages as well as performance of the propulsion systems themselves after assembly.

\section{Conclusion}

\section{VI.A. Summary}

We have proposed a lean, fast mission to Mars using current or readily-achievable technologies to be planned for the years 2034, 2035, and/or 2037. We propose a "lean" mission with the intent of pursuing minimum weight, power, and volume required for a crew transit vehicle, as well as pursuing a minimal mission profile and set of objectives for the very first mission to Mars that would hope to minimize cost. We propose a "fast" mission with the intent of controlling to a reduced round trip time so as to be able to have a "lean" transit that requires minimal supplies, but also with the intent of promoting reasonable risk controls for a short trip that otherwise would be unsatisfactory and also would inflate the crew transit vehicle weight. Lastly, we propose the use of 'current or readily-achievable technologies' in 
order to promote a mission to Mars that could be reasonably achieved by the 2030s, but also promotes stretching our technologies in smaller, more achievable steps while simultaneously realizing the next giant leap in deep space travel and exploration.

We expect that the number of launches can be significantly reduced from these very first calculations that have been presented based on results from later sensitivity studies, and that launch efficiencies can also be readily employed to utilize ELVs for lighter-weight payloads not requiring the full SLS launch capability. To be certain, however, we also note that there are technological and operational challenges to be addressed with this approach. Specifically, these include identifying a feasible operational launch cadence, as well as proving the use of automated rendezvous and docking (AR\&D) to perform in-orbit assembly and expanding our technology for controlling in-orbit cryogenic boil-off for durations on the order of a few months. We also expect that several more in-depth assessments will be conducted in follow-on sensitivity studies to flesh out more information to support feasibility checks and balances.

Finally, we submit that our recommendation to conduct a first Mars expedition in the form of a one-year round trip proposed for the years 2034, 2035, and/or 2037, is providing a very opportune time-frame for GCR risk controls among other risk controls. We believe that the benefits offered by controlling the round-trip duration to approximately one year serve to control risks as a whole as well as promote a minimal crew transit vehicle weight, which in turn supports the feasibility of this short duration. We have shown that all of these features are paramount in achieving this balance, and that they must be viewed as interdependent factors to achieve an optimized overall design and mission architecture, and not addressed as individual elements to be optimized separately. In so doing, we offer a wholly-integrated concept that promotes a first mission to Mars for the 2030s.

\section{VI.B. Future Work}

The next stage of this study will include sensitivity analysis to several of the decision parameters. This analysis may be accomplished by mining and processing the existing trajectory data set. First, we will vary the number of launches to between 8 and 12 SLS vehicles and see what flight times can be achieved for each propulsion system and number of launches combination. Second, we will further analyze sensitivity of the number of required launches to the length of stay at Mars. Third, we determine the sensitivity of the number of launches to the mass of the DSH in finer resolution than we have done so far. Also, the ADL team will examine the sensitivity of IMLEO to a range of cryogenic boil-off rates.

In addition to sensitivity analysis, we will examine in more detail the Mars orbit and landing component of the missions (Phase 3). We will characterize the $\delta v$ necessary to transfer from the arrival orbit about Mars to the departure orbit, the trajectory and hardware 
for the $\mathrm{D} / \mathrm{AV}$, and develop a conops for Phase 3 .

Finally, we will consider alternative mission architectures. In the first of these architectures, we will replace the pre-position stack (Phase 1) with a large-scale solar-electric propulsion (SEP) or nuclear-electric propulsion (NEP) transfer vehicle. SEP or NEP are alternatives to chemical propulsion which can often deliver more payload to the destination for a smaller IMLEO at the cost of a longer flight time. While neither SEP or NEP is a mature technology on the scale of this study, either could significantly reduce the number of launches. We will use SEP or NEP only for the Phase 1, which is not time-sensitive. The SEP or NEP system will also be used to position the Phase 1 vehicle into the Phase 4 departure orbit, which will the Phase 3 rendezvous problem easier. We will continue to use chemical propulsion for the crewed vehicle (Phases 2 and 4 ) because flight time is more important than mass fraction for those phases.

The second alternative architecture will be an uncrewed version of the current Fast Mars architecture which would demonstrate each phase of the mission, especially the critical Phase 3 rendezvous. The third and final alternative architecture will be a crewed round trip to Mars with an uncrewed landing. The crew would instead control a telerobotic surface mission.

\section{Acknowledgments}

The authors would like to thank several people who made significant contributions to this study. Bruce Campbell, who at the time of the study was the manager of the Integrated Design Center (IDC) at GSFC, led the study. Bob Estes and Sara Riall, both of GSFC, performed the propulsion and mechanical systems designs, respectively.

We acknowledge, with great appreciation, the guidance and efforts from Francis $\mathrm{Cu}-$ cinotta, Neal Zapp, Edward Semones, Janet Barzilla, Steven Koontz, Bobbie Swan, William Atwell, and Kristina Rojdev as noteworthy JSC contributors for radiation information, support, and analysis. We want to thank David Smitherman and his team of contributors at the MSFC Advanced Concepts Office for their well-developed ISS-Derived vehicle concept and their outstanding support. We also acknowledge the significant contributions of Joe Chambliss, Jordan Metcalf, and Johnathan Dory for their outstanding, instrumental efforts in organizing the technical interchange meetings on the topics of ECLSS and Human Health and Performance. We also express appreciation for the tremendous safety and risk analysis support from Melissa Flores, Scott Winter, and Theresa Castillo. A note of personal thanks to Nanette Faget, who supported this effort and the lead author in the capacity of branch chief and all-around encourager.

We also recognize two special JSC contributions - the wise guidance and support of Alvin Drew, who was the JSC Project Manager for the DSH at the time of the study, as well as the 
outstanding and steadfast administrative support from Gayla Lind-Olsen, without whom, these studies could not have been accomplished.

\section{References}

${ }^{1}$ Drake, B. G., editor, Human Exploration of Mars: Design Reference Architecture 5.0 Addendum, NASA/SP-2009-566-ADD, 2009, downloadable from URL http://www.nasa.gov/pdf/373667main NASA-SP-2009-566-ADD. pdf.

${ }^{2}$ Bailey, L., "FY 2012 Strategic Implementation Plan for the First Deep Space Command Post," Tech. rep., NASA JSC, January 2012.

${ }^{3}$ Bailey, L., "Proposal for a Lean, Current-TRL-Favored Architecture Concept of a Mars Excursion Mission Assessing an ISS-Derived Deep Space Habitat,” Tech. rep., NASA JSC, July 2013.

${ }^{4}$ Folta, D. C., Vaughn, F. J., Westmeyer, P. A., Rawitscher, G. S., Bordi, F., "Enabling Exploration Missions Now: Applications of On-Orbit Staging," Proceedings of the AAS/AIAA Astrodynamics Specialist Conference, Lake Tahoe, CA, August 7-11, 2005. Paper AAS 05-273.

${ }^{5}$ Folta, D. C., Barbee, B. W., Englander, J., Vaughn, F., Lin, T. Y., "Optimal Round-Trip Trajectories for Short Duration Mars Missions," Proceedings of the AAS/AIAA Astrodynamics Specialist Conference, Hilton Head, SC, August 11-15, 2013. Paper AAS 13-808.

${ }^{6}$ Smitherman, D. t., "Deep Space Habitat Configurations Based on International Space Station Systems," Tech. rep., NASA MSFC, December 2011.

${ }^{7}$ Cucinotta, F. A., Myung-Hee, K. Y., and Cappell, L., "Space Radiation Cancer Risk Projections and Uncertainties - 2010," Tech. Rep. TP-2011-216155, NASA, 2011.

8 "NASA Space Flight Human System Standard," Tech. Rep. NASA-STD-3001, NASA, 2007, https : //standards.nasa.gov/documents/detail/3315622.

9 "NASA Space Flight Human System Standard," Tech. Rep. NASA-STD-3001, NASA, 2007, https : //standards.nasa.gov/documents/detail/3315785.

${ }^{10}$ Semones, E., "Radiation Limits for Astronauts," Tech. rep., NASA JSC, December 2011.

${ }^{11}$ Koontz, S. L., "GCR Shielding Mass: Materials Selection and Programmatic Impacts," Tech. rep., NASA JSC, January 2012.

${ }^{12}$ Barzilla, J., "Node 2 'Hub and Spoke' Design Radiation Analysis," Tech. rep., NASA JSC, May 2012.

${ }^{13}$ Barzilla, J., "Incorporating Radiation Shielding into Vehicle Design for exo-LEO Missions," Tech. rep., NASA JSC, January 2012.

${ }^{14}$ Castillo, T., "ISS Probabilistic Risk Assessment (PRA) Human Reliability Analysis (HRA)," Tech. rep., NASA JSC, March 2012.

${ }^{15}$ Prussing, J. and Conway, B., Orbital Mechanics, Oxford University Press, New York, 1993.

${ }^{16}$ Wiesel, W. E., Spaceflight Dynamics, Irwin/McGraw-Hill, 2nd ed., 1997.

${ }^{17}$ Curtis, H. D., Orbital Mechanics for Engineering Students, Elsevier, 2nd ed., 2009.

${ }^{18}$ Barbee, B. W., Esposito, T., Piñon, E. III, Hur-Diaz, S., Mink, R. G., and Adamo, D. R., "A Comprehensive Ongoing Survey of the Near-Earth Asteroid Population for Human Mission Accessibility," Proceedings of the AIAA/AAS Guidance, Navigation, and Control Conference, Toronto, Ontario, Canada, 2-5 August 2010, Paper 2010-8368. 
${ }^{19}$ Barbee, B. W., Mink, R. G., Adamo, D. R., and Alberding, C. M., "Methodology and Results of the Near-Earth Object (NEO) Human Space Flight (HSF) Accessible Targets Study (NHATS)," Advances in the Astronautical Sciences, Vol. 142, 2011, pp. 613-632, also AAS/AIAA Paper AAS 11-444, AAS/AIAA Astrodynamics Specialist Conference, Girdwood, Alaska, July 31 - August 4, 2011.

${ }^{20}$ Barbee, B.W., Abell, P.A., Adamo, D. R., Alberding, C. M., Mazanek, D. D., Johnson, L. N., Yeomans, D. K., Chodas, P. W., Chamberlin, A. B., Friedensen, V. P., "The Near-Earth Object Human Space Flight Accessible Targets Study: An Ongoing Effort to Identify Near-Earth Asteroid Destinations for Human Explorers," Proceedings of the IAA Planetary Defense Conference 2013, Flagstaff, AZ, April 15-19, 2013.

${ }^{21}$ Englander, J. A., Conway, B. A., and Williams, T., "Automated Mission Planning via Evolutionary Algorithms," Journal of Guidance, Control, and Dynamics, Vol. 35, No. 6, November-December 2012, pp. 613-632, DOI: 10.2514/1.54101.

${ }^{22}$ Englander, J. A., Conway, B. A., Williams, T., "Automated Interplanetary Mission Planning," AAS/AIAA Astrodynamics Specialist Conference, Minneapolis, MN, August 2012.

${ }^{23}$ Englander, J. A., Automated Trajectory Planning for Multiple-Flyby Interplanetary Missions, Ph.D. thesis, University of Illinois at Urbana-Champaign, April 2013.

${ }^{24}$ Vinkó, T. and Izzo, D., "Global Optimisation Heuristics and Test Problems for Preliminary Spacecraft Trajectory Design," Tech. Rep. GOHTPPSTD, European Space Agency, the Advanced Concepts Team, 2008, Available on line at www.esa.int/act.

25 "Global Trajectory Optimization Problem Database," October 2012, http://www.esa.int/gsp/ACT/ inf/op/globopt.htm.

${ }^{26}$ Deb, K., Pratap, A., Agarwal, S., and Meyarivan, T., "A fast and elitist multiobjective genetic algorithm: NSGA-II," Evolutionary Computation, IEEE Transactions on, Vol. 6, No. 2, 2002, pp. $182-197$.

${ }^{27}$ Bailey, L. J. and Folta, D. t., "Fast Mars Transfer using On-Orbit Staging, Proposal for a Lean, Current-TRL-Favored Mars Excursion Concept," Tech. rep., NASA JSC and NASA GSFC, June 2013. 


\title{
A PROPOSAL FOR A LEAN, FAST MARS ROUND-TRIP MISSION ARCHITECTURE: USING CURRENT TECHNOLOGIES FOR A HUMAN MISSION TO MARS IN THE 2030s
}

\author{
Lora Bailey, David Foltał Brent W. Barbee, Frank Vaughn, Bruce Campbell, \\ Harley Thronson, Jacob Englander,** and Tzu Yu Lin ${ }^{\dagger \dagger}$
}

We present a lean-minded, fast-transfer mission strategy and architecture concept for a first human mission to Mars that deliberately utilizes a current-technology-favored approach by means of introducing and quantitatively defining two pivotal parameters: 1) an end-to-end Mars mission duration of approximately one year, and 2) a deep space habitat of approximately 40-50 metric tons. These parameters are identified and introduced by a 2012 deep space habitat study conducted at the NASA Johnson Space Center (JSC) that focused on a subset of recognized high-engineering-risk factors that may otherwise inhibit or encumber remote space travel to destinations such as Mars or near-Earth asteroids (NEAs). ${ }^{1}$ Additional constraints in the study favoring current technology and a lean-minded (very short) surface stay on Mars are shown to offer such Mars mission opportunities in the 2030s, enabled by a combination of on-orbit staging, ${ }^{2}$ mission element pre-positioning, and unique round-trip trajectories identified by state-of-the-art astrodynamics algorithms. ${ }^{3,4}$
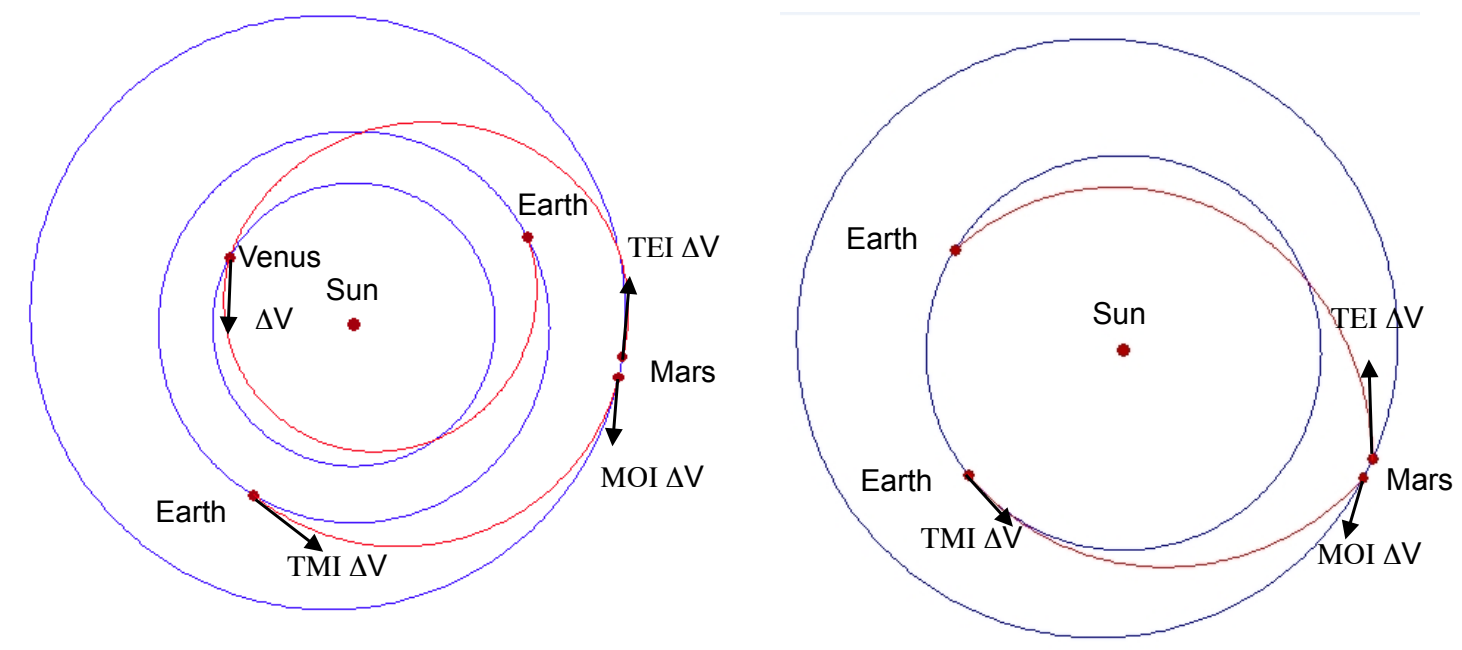

(a) Optimal one year duration round-trip trajectory to (b) Optimal one year duration round-trip trajectory to Mars with a Venus gravity assist on the Earth-return por- Mars without a Venus gravity assist. tion of the trajectory.

Figure 1. Exemplar optimal one year duration round-trip trajectories to Mars.

This astrodynamics analysis, requested by JSC as part of the Deep Space Habitat risk-reduction study, was performed by the NASA Goddard Space Flight Center (GSFC) and utilizes a Fast Mars Transfer (FMT) approach which had previously been developed in an early study by GSFC in 2004-2005. The new 2012 analysis employed the use of parameters defined by the JSC study in order to assess the feasibility of a round

\footnotetext{
*Aerospace Engineer, NASA/JSC, Code EA32, 2101 NASA Parkway, Houston, TX, 77058, USA.

$\dagger$ Aerospace Engineer, NASA/GSFC, Code 595, 8800 Greenbelt Road, Greenbelt, MD 20771, USA.

$¥$ Aerospace Engineer, NASA/GSFC, Code 595, 8800 Greenbelt Road, Greenbelt, MD 20771, USA.

$\S$ Aerospace Engineer, NASA/GSFC, Code 595, 8800 Greenbelt Road, Greenbelt, MD 20771, USA.

IIntegrated Design Center (IDC) Manager, NASA/GSFC, Code 500, 8800 Greenbelt Road, Greenbelt, MD 20771, USA.

" Senior Scientist for Advanced Concepts, NASA/GSFC, Code 660, 8800 Greenbelt Road, Greenbelt, MD 20771, USA.

**Aerospace Engineer, NASA/GSFC, Code 595, 8800 Greenbelt Road, Greenbelt, MD 20771, USA.

${ }^{\dagger \dagger}$ Graduate Student, Mechanical and Aerospace Engineering Department, University of Florida, Gainesville, FL, 32611, USA.
} 
trip mission using only current chemical propulsion technology that would offer a brief (approximately twoday) human surface landing on Mars within a total round-trip mission duration of approximately one year using a deep space habitat of approximately 50 metric tons. Identification of notionally feasible and optimal (minimum Initial Mass in Low Earth Orbit (IMLEO)) trajectory solutions for these missions was enabled by a combination of GSFC algorithms for optimal on-orbit staging, pre-positioning of assets at Mars, and state-of-the-art astrodynamics algorithms for comprehensive identification of optimal round-trip trajectory solutions with and without Venus gravity assists; see Figures 1(a) and 1(b), respectively. The four-stage propulsion module for Earth return modeled in the on-orbit staging algorithms is pre-positioned in Mars orbit and subsequently docked with the crew's deep space habitat upon the habitat's arrival in Mars orbit at a later date. The results of this analysis show that one-year duration short-stay Mars mission opportunities using chemical propulsion are available in the mid-2030s and for which the predicted number of 120 metric ton to LEO heavy-lift launches is in the range of 10-12. The results also show that, when only chemical propulsion is used, this unique mission opportunity window opens briefly in the mid-2030s and does not open again for approximately 17 years.

The purpose of our effort is to provide a pilot study of a lean-minded Mars mission, with the intention of promoting affordability and making a best effort to utilize known, familiar elements - including International Space Station modules and systems when possible - as well as conventional propulsion technology (e.g. hypergolic bi-propellant and/or cryogenic propellant with appropriate storage). In doing so, it is our hopeful expectation that 1) key risks can be mitigated, and that 2) this will greatly diminish the delay in initiating such a venture.

The full architectural concept includes an evaluation of a possible gradual build-up to the previouslydescribed short duration human landing on Mars. This build-up includes concepts for an initial non-crewed round-trip test flight to Mars featuring an autonomous sample return, followed by a preliminary short duration human mission to Mars orbit during which a sample collection experiment would be conducted tele-robotically. A Venus flyby during the Earth-return leg of the journey is also considered, weighing a slight mission mass reduction and diversity of mission destinations against close approaches to the Sun.

\section{References}

\footnotetext{
${ }^{1}$ Bailey, L., "Radiation Studies for a Long Duration Deep Space Habitat Transit," Future In-Space Operations (FISO) colloquia [online database], http://spirit.as.utexas.edu/ fiso/telecon.htm [cited 31 January 2013].

${ }^{2}$ Folta, D. C., Vaughn, F. J., Westmeyer, P. A., Rawitscher, G. S., and Bordi, F., "Enabling Exploration Missions Now: Applications of On-Orbit Staging," Proceedings of the AAS/AIAA Astrodynamics Specialist Conference, 2005, Paper AAS 05-273.

${ }^{3}$ Barbee, B. W., Mink, R. G., Adamo, D. R., and Alberding, C. M., "Methodology and Results of the Near-Earth Object (NEO) Human Space Flight (HSF) Accessible Targets Study (NHATS)," Advances in the Astronautical Sciences, Vol. 142, 2011, pp. 613-632, also AAS/AIAA Paper AAS 11-444, AAS/AIAA Astrodynamics Specialist Conference, Girdwood, Alaska, July 31 - August 4, 2011.

${ }^{4}$ Englander, J. A., Conway, B. A., and Williams, T., "Automated Mission Planning via Evolutionary Algorithms," Journal of Guidance, Control, and Dynamics, Vol. 35, No. 6, November-December 2012, pp. 613-632, DOI: 10.2514/1.54101.
} 